\title{
Second-order scalar wave field modeling with a first-order perfectly matched layer
}

\author{
Xiaoyu Zhang, Dong Zhang, Qiong Chen, and Yan Yang \\ School of Physics and Technology, Wuhan University, Wuhan, Hubei, China
}

Correspondence: Dong Zhang (dongz@whu.edu.cn)

Received: 29 May 2018 - Discussion started: 11 June 2018

Revised: 21 September 2018 - Accepted: 23 October 2018 - Published: 7 November 2018

\begin{abstract}
The forward modeling of a scalar wave equation plays an important role in the numerical geophysical computations. The finite-difference algorithm in the form of a second-order wave equation is one of the commonly used forward numerical algorithms. This algorithm is simple and is easy to implement based on the conventional grid. In order to ensure the accuracy of the calculation, absorption layers should be introduced around the computational area to suppress the wave reflection caused by the artificial boundary. For boundary absorption conditions, a perfectly matched layer is one of the most effective algorithms. However, the traditional perfectly matched layer algorithm is calculated using a staggered grid based on the first-order wave equation, which is difficult to directly integrate into a conventionalgrid finite-difference algorithm based on the second-order wave equation. Although a perfectly matched layer algorithm based on the second-order equation can be derived, the formula is rather complex and intermediate variables need to be introduced, which makes it hard to implement. In this paper, we present a simple and efficient algorithm to match the variables at the boundaries between the computational area and the absorbing boundary area. This new boundarymatched method can integrate the traditional staggered-grid perfectly matched layer algorithm and the conventional-grid finite-difference algorithm without formula transformations, and it can ensure the accuracy of finite-difference forward modeling in the computational area. In order to verify the validity of our method, we used several models to carry out numerical simulation experiments. The comparison between the simulation results of our new boundary-matched algorithm and other boundary absorption algorithms shows that our proposed method suppresses the reflection of the artificial boundaries better and has a higher computational efficiency.
\end{abstract}

\section{Introduction}

Modeling of a seismic wave field is accomplished by simulating the pattern of the seismic waves as they propagate through various geologic media and computing the simulated measurements at observation points on the Earth's surface or underground, given that the underground medium's structure and the relevant physical parameters are known. Numerical modeling of a seismic wave field is an important tool for seismic data processing and interpretation and for geodynamic studies of the Earth's interior. In recent years, many full waveform inversion methods have been widely proposed and applied to seismic exploration. In the waveform inversion process, wave field modeling is one of the key algorithms because it must be performed first to obtain the predicted wave field that is used to compute the residual errors between the predicted and the actual wave field records. In addition, the information provided by the residual errors, which is required for refinement of the initial model, is actually calculated by a modeling algorithm that uses the residual errors as virtual sources. After many iterations of the above processes, an optimized approximate model of the underground medium can be acquired. Numerical modeling of a wave field will be executed thousands of times throughout the waveform inversion process, so a wave field modeling algorithm is crucial in many ways when performing a waveform inversion algorithm, such as for computational precision, speed, and storage requirements.

The main numerical techniques for seismic wave field modeling include the finite-element method (Marfurt, 1984; Yang et al., 2008), the pseudo-spectral method (Kreiss and Oliger, 1972; Dan and Baysal, 1982), and the finitedifference method (Kelly et al., 2012; Virieux, 1984; Yang et al., 2002; Moczo et al., 2007; Zhang et al., 2013). Due to 
(a)

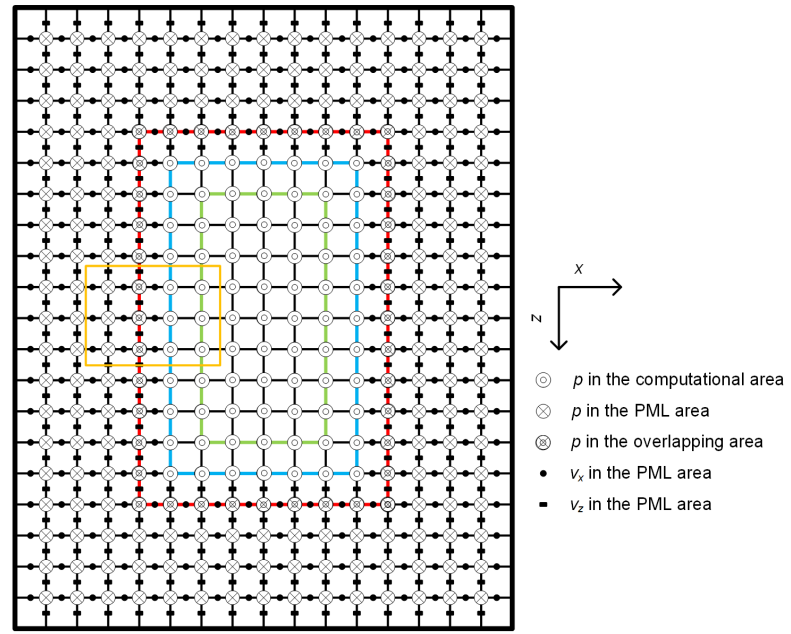

(b)

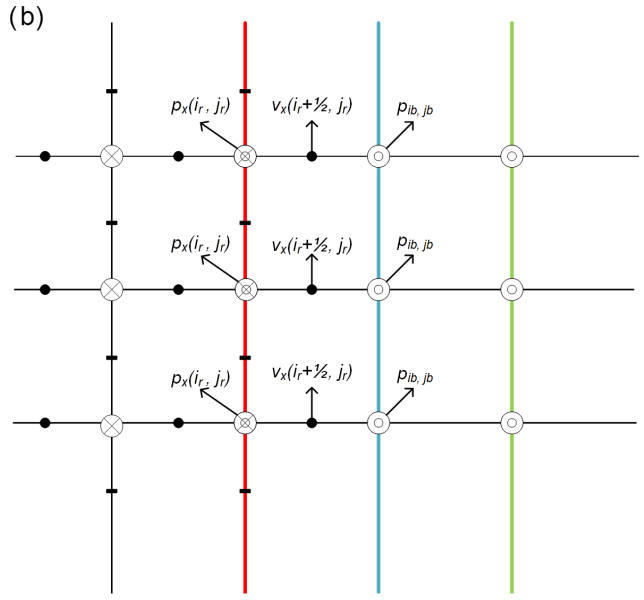

Figure 1. Schematic of our method: (a) the entire region and (b) partial enlargement of the yellow rectangle in panel (a).

its easy implementation and the satisfactory compromise between accuracy and efficiency, the finite-difference method is the preferred method. For a comprehensive overview of applications of the finite-difference methods, see Moczo et al. (2014). Over the last several decades, many studies have focused on determining the coefficients of the finitedifference method and designing computational templates (Li et al., 2017).

According to the formulation of the wave equations, the finite-difference methods can be implemented based on the first-order velocity-stress equations or the second-order displacement equations, which lead to different computational templates. A staggered grid (SG) is usually set up for the first-order wave equations and has been widely used with the acoustic and elastic wave equations (Virieux, 1984, 1986; Moczo et al., 2014; Madariaga, 1976; Gold et al., 1997; Saenger et al., 2000; O’Brien, 2010). Many methods of optimizing the differential coefficients, based on a SG, have been proposed to increase the accuracy of the numerical solution, such as the time-space domain dispersion-relation-
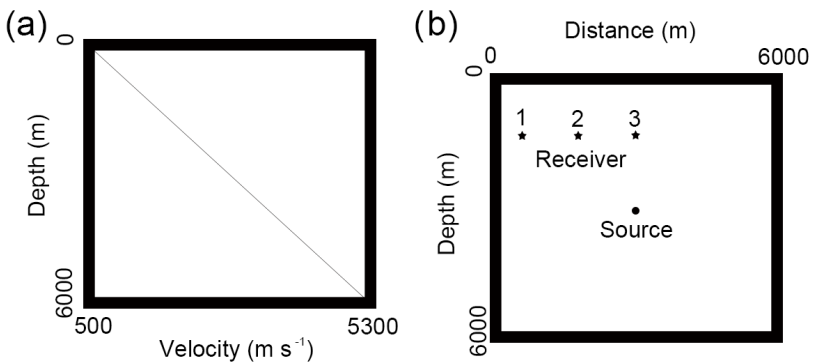

Figure 2. (a) Velocity profiles in depth and (b) distribution of source and receivers.

based method (Liu and Sen, 2011), the simulated annealing algorithm (Zhang and Yao, 2013), and the least-squares method (Yang et al., 2015). However, a conventional grid (CG) is often directly obtained from the second-order wave equation. These methods include the central scheme (Alford et al., 1974; Igel et al., 1995), the high-order compact finitedifference method (Fornberg, 1990), the Lax-Wendroff correction (LWC) scheme (Lax and Wendroff, 1964; Dablain, 1986; Blanch and Robertsson, 2010), the nearly analytical discrete method (Yang et al., 2003), and the nearly analytical central difference method (Yang et al., 2012).

The algorithm design of the CG scheme is easier to use than that of the SG scheme because the variable definition is uniform throughout the grid. However, it is hard to determine which of the two schemes is more accurate and efficient. Although the SG scheme has sometimes been regarded as more precise than the CG scheme (Huang and Dong, 2009), there is also some theoretical and experimental proof in the literature that does not support this proposition. Moczo et al. (2011) compared the accuracy of the different finite-difference schemes with respect to the $P$-wave to $S$-wave speed ratio using theoretical analysis and numerical experiments. Their investigation showed that the relative local errors of the CG scheme are almost equal to those of the SG scheme when modeling planar $S$ waves propagating in an unbounded homogeneous elastic isotropic medium with a low $P$-wave to $S$-wave velocity ratio $\left(V_{p} / V_{s}=1.42\right)$. They showed that only at higher $P$-wave to $S$-wave velocity ratios $\left(V_{p} / V_{S}=5,10\right)$ will the relative local error of the CG scheme increase faster than that of the SG scheme, but the difference in the relative local errors of the two schemes will decrease when using a higher-order spatial scheme, i.e., from second-order to fourth-order in space. Moczo et al. (2011) also showed that the insufficient accuracy of the CG scheme at higher $P$-wave to $S$-wave speed ratios can be compensated for by using a higher spatial sampling ratio, i.e., a smaller grid size. This means that a CG scheme with a sufficiently small grid size will be as precise as the SG scheme or better, even if the $P$-wave to $S$-wave speed ratio is high. The computational cost of the SG scheme is significantly higher than that of an equal-sized CG scheme, as two variables (velocity 

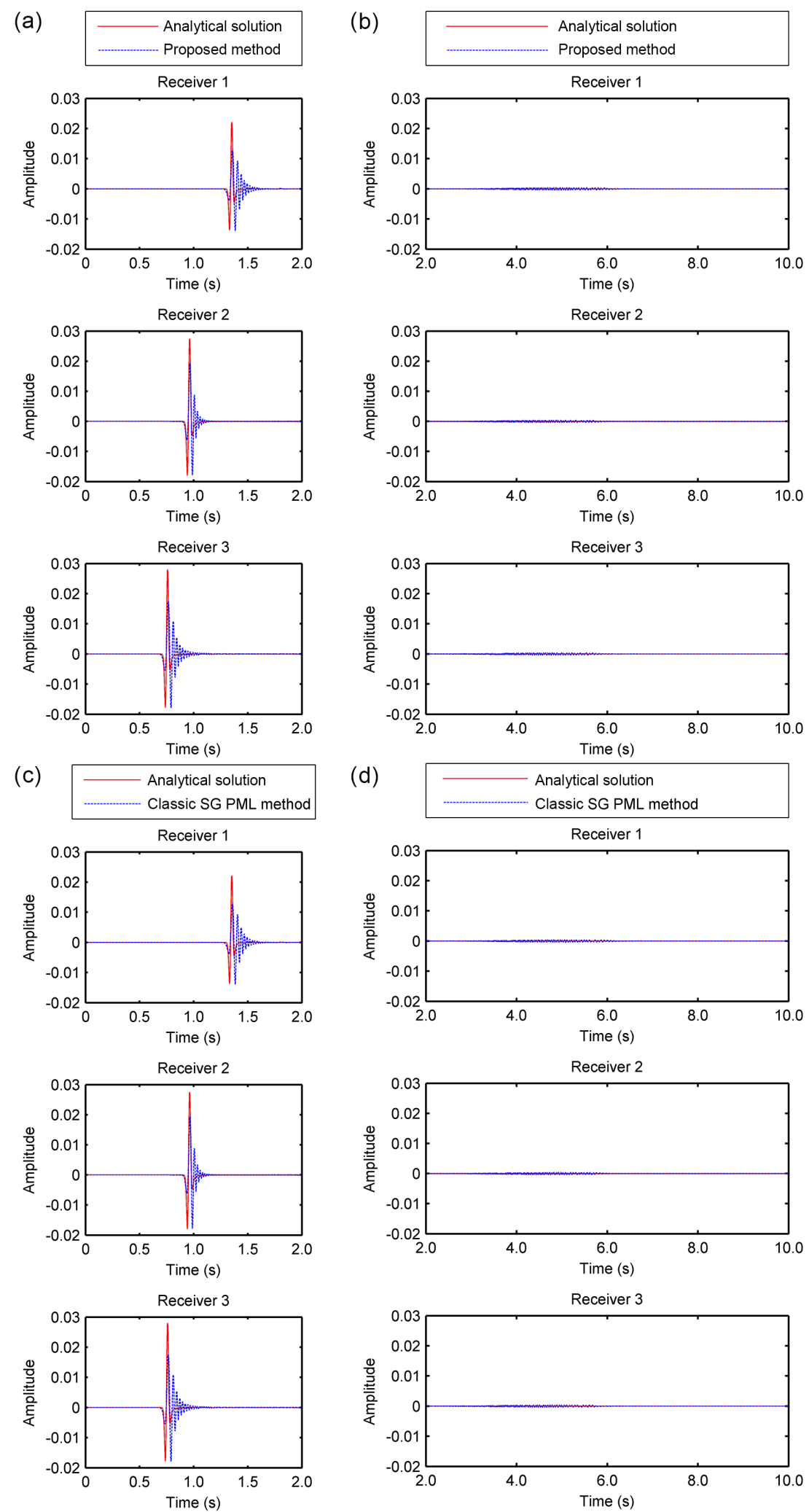

Figure 3. Comparison of the analytical solution (red solid line) with the proposed (second-order conventional grid (CG) scheme) and classic staggered-grid (SG) perfectly matched layer (PML) methods (second-order SG scheme) (blue dotted line) at different receivers, $d=12 \mathrm{~m}$. (a) Proposed method at receivers 1, 2, and 3 for the first $2 \mathrm{~s}$; (b) proposed method at receivers 1, 2, and 3 after $2 \mathrm{~s}$; (c) classic SG PML method at receivers 1, 2, and 3 for the first $2 \mathrm{~s}$; (d) classic SG PML method at receivers 1, 2, and 3 after $2 \mathrm{~s}$. 
(a)
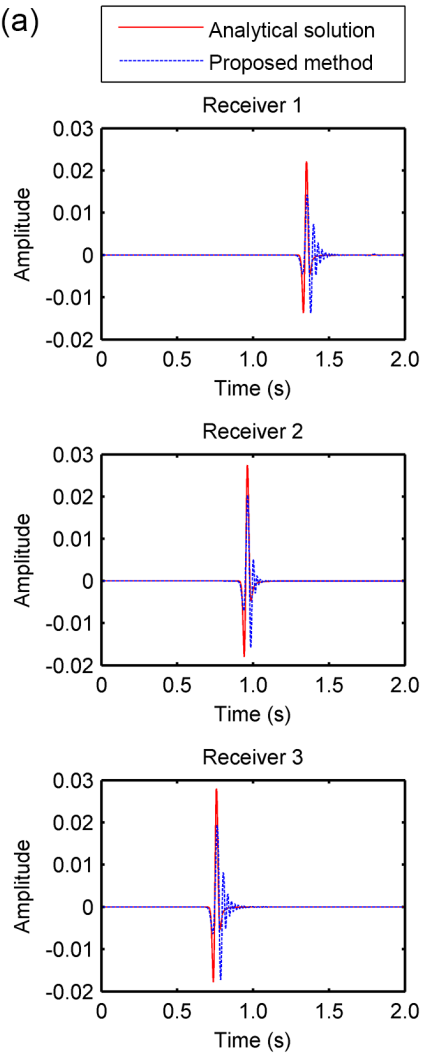

(c)
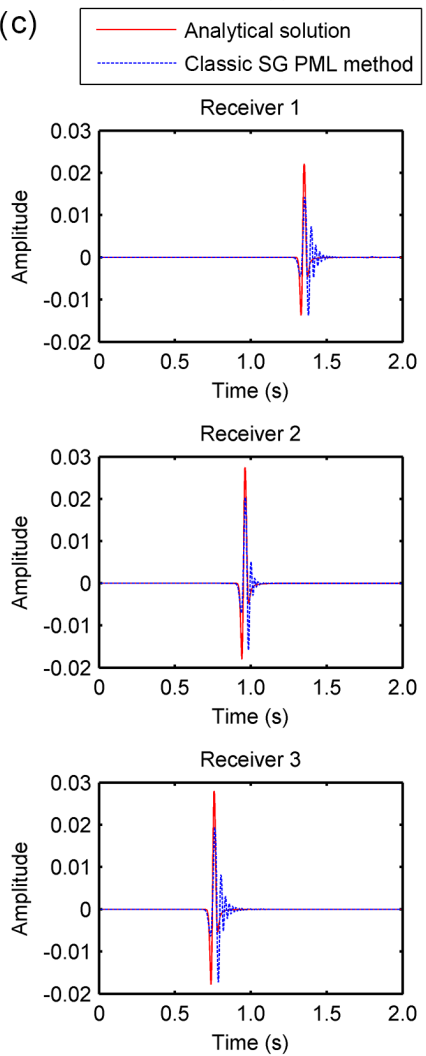

(b)

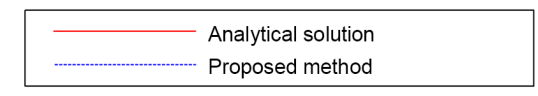

Receiver 1

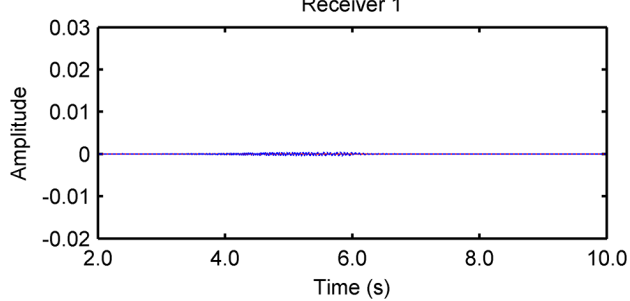

Receiver 2

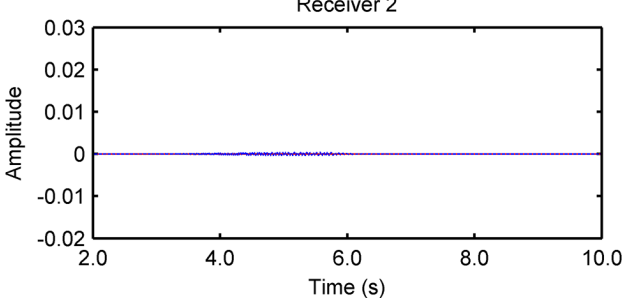

Receiver 3

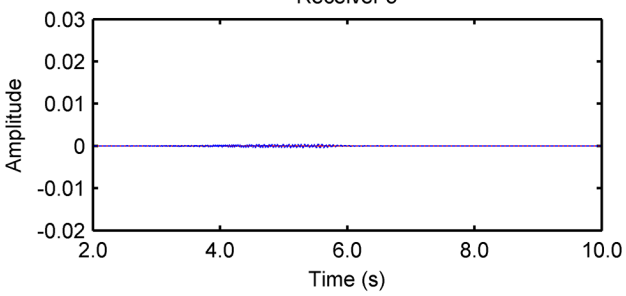

(d)

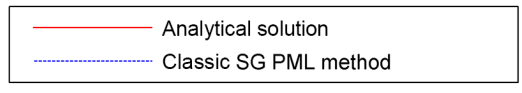

Receiver 1

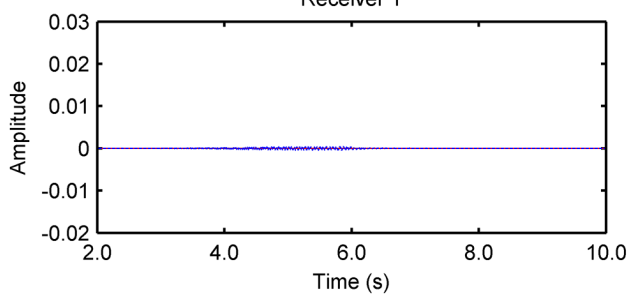

Receiver 2

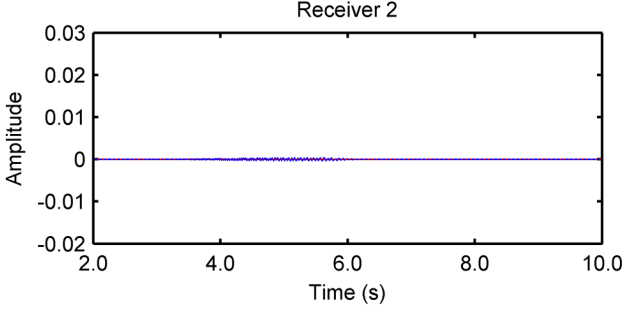

Receiver 3

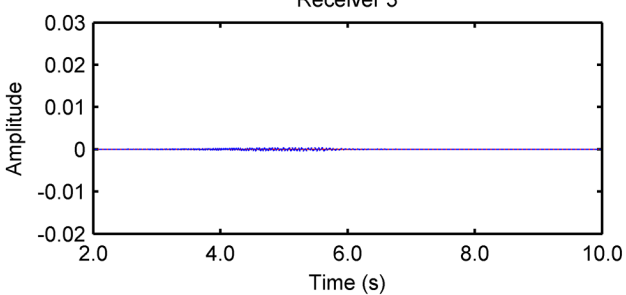

Figure 4. $d=10 \mathrm{~m}$; the rest is the same as in Fig. 3 . 
(a)
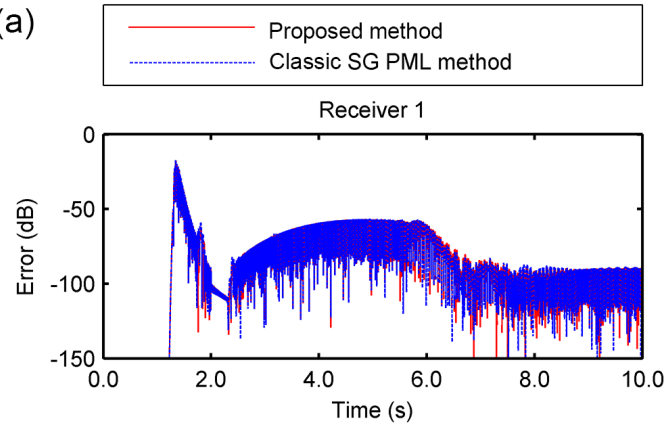

Receiver 2
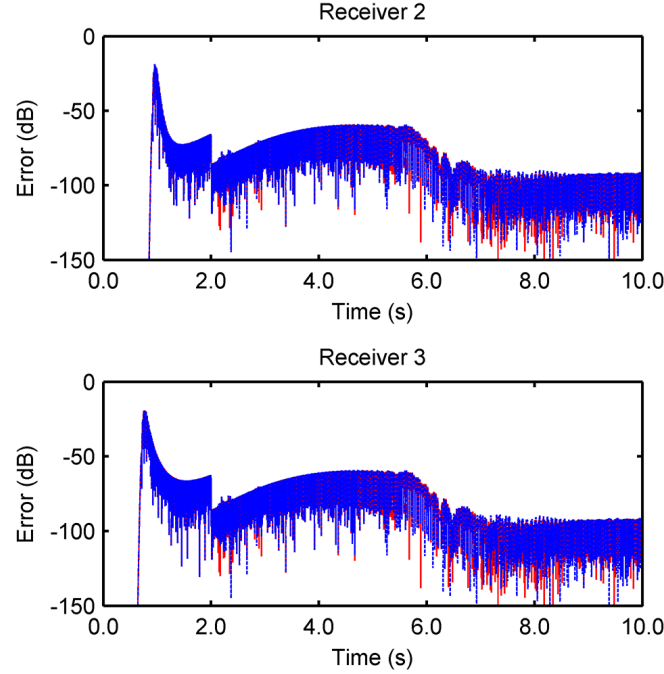

(b)
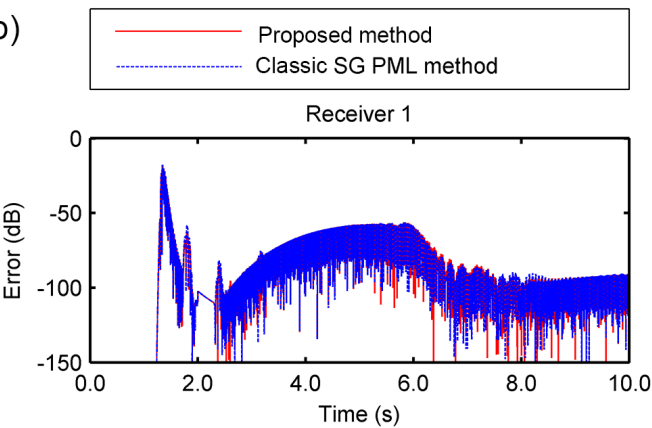

Receiver 2

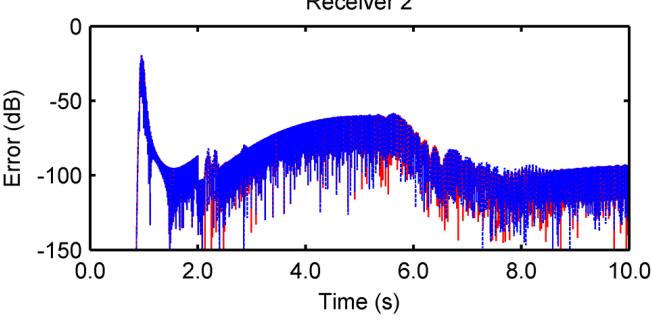

Receiver 3

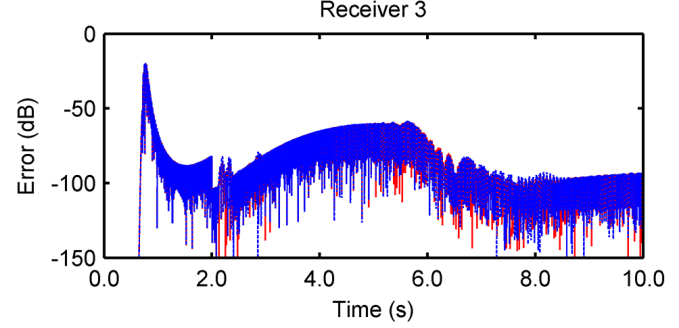

Figure 5. Comparison of the relative errors between the analytical solutions and the proposed method (second-order CG scheme) (red solid line) or the classic SG PML method (second-order SG scheme) (blue dotted line) at different receivers and different grid spacings. (a) $d=12 \mathrm{~m}$, receivers 1,2 , and 3; (b) $d=10 \mathrm{~m}$, receivers 1,2 , and 3 .

and stress) have to be calculated in the SG scheme and only one variable (displacement) have to be computed in the CG scheme.

Reflection from the artificial boundaries introduced by the limited computational area is another numerical source of error. Over the past 30 years, many techniques have been developed for boundary processing: paraxial conditions (Clayton and Engquist, 1977; Reynolds, 1978; Higdon, 2012), the sponge boundary (Cerjan et al., 1985; Sochacki et al., 1987), the perfectly matched layer (PML) (Berenger, 1994), and the hybrid absorbing boundary conditions (hybrid ABC) (Ren and Liu, 2012). Among these, the PML is one of the most efficient and most commonly used methods. The PML was first introduced for boundary processing of electromagnetic wave equation modeling, after which, it was applied to the elastic-dynamic problem (Chew and Liu, 1996) and acoustic simulations (Liu and Tao, 1998). Many modified versions of the PML, such as the convolutional PML (Komatitsch and Martin, 2007), were subsequently proposed. Gao et al. (2017) compared most of the typical artificial absorbing boundary processing approaches for use with acoustic wave equations and came to the conclusion that a 20-layer PML is ideal for most practical applications using general size models, even in the presence of strong nearly grazing waves, which demonstrates the high performance and efficiency of the PML approach.

In the field of real wave field simulation, most researchers are devoted to unifying the format of the boundary processing algorithm and the wave equation within the computational region. The classic PML is naturally formulated based on the first-order wave equations for velocity and stress (Collino and Tsogka, 1998), which has proven to be very efficient. It is easy to integrate PML boundary processing into a SG finite-difference algorithm. So, some scholars use the SG scheme in the computational region to match the PML equations, while for many CG-based schemes, they need to adopt other boundary processing methods, such as the hybrid ABC method. However, in recent years, some scholars have also made efforts to formulate a PML for a second-order system to match the second-order wave equation. Komatitsch and Tromp (2003) reformulated the classic PML conditions in order to use it with numerical schemes that are based on the elastic wave equation written as a second-order system with displacement. Grote and Sim (2010) proposed a PML formu- 

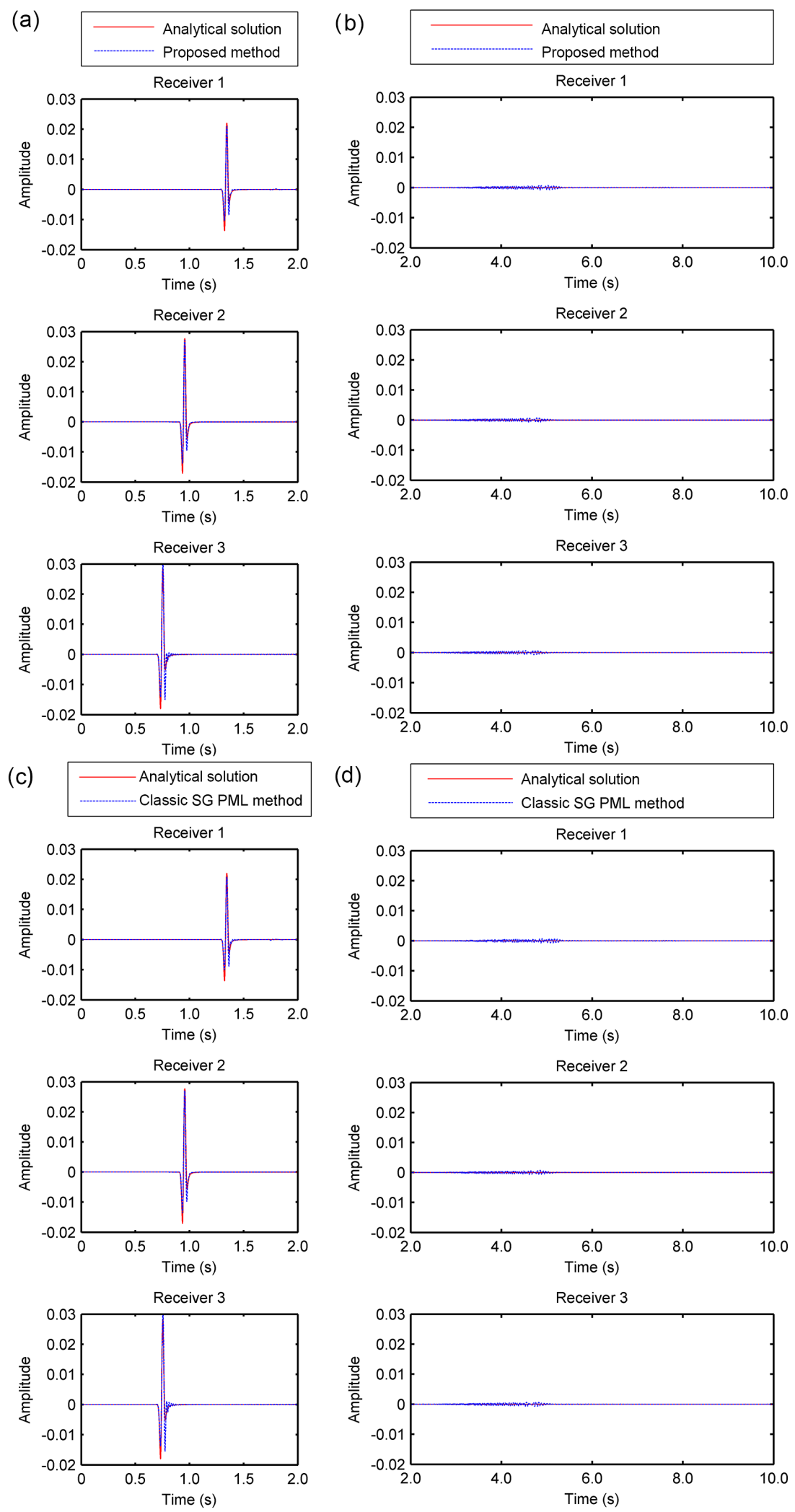

Figure 6. Comparison of the analytical solution (red solid line) with the proposed (fourth-order CG scheme) and classic SG PML methods (fourth-order SG scheme) (blue dotted line) at different receivers and $d=12 \mathrm{~m}$. (a) Proposed method at receivers 1, 2, and 3 during the first $2 \mathrm{~s}$; (b) proposed method at receivers 1, 2, and 3 after $2 \mathrm{~s}$; (c) classic SG PML method at receivers 1, 2, and 3 during the first $2 \mathrm{~s}$; (d) classic SG PML method at receivers 1,2 , and 3 after $2 \mathrm{~s}$. 
(a)
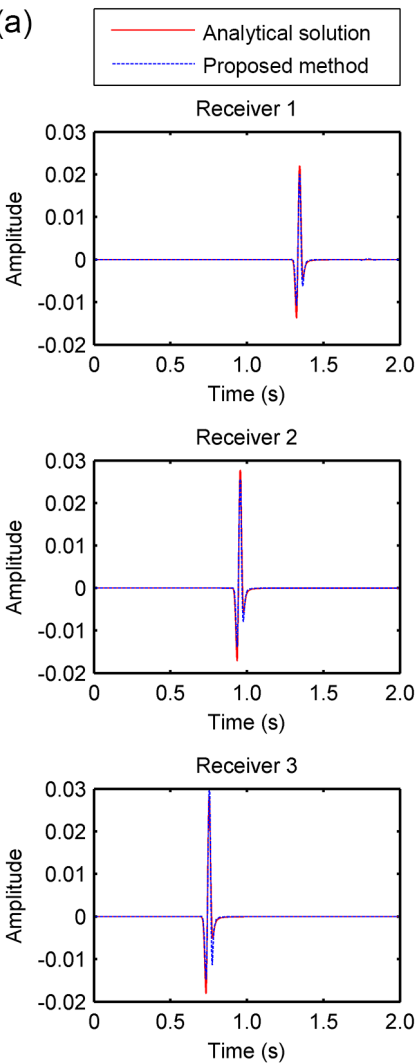

(c)
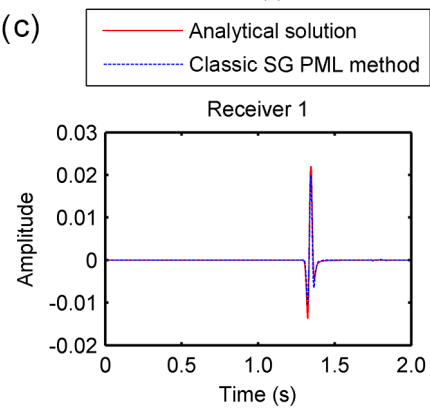

Receiver 2
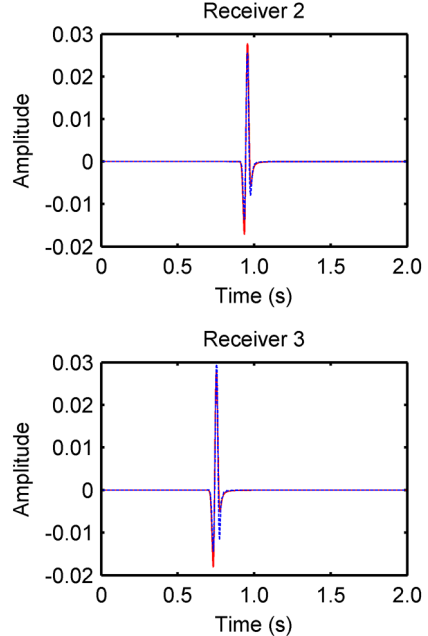

(b)

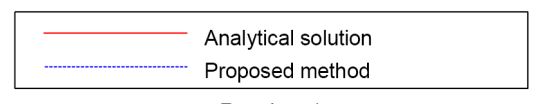

Receiver 1

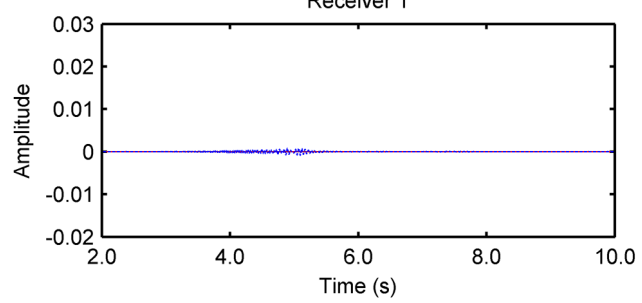

Receiver 2

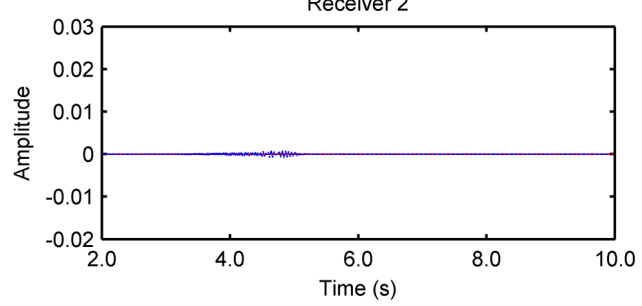

Receiver 3

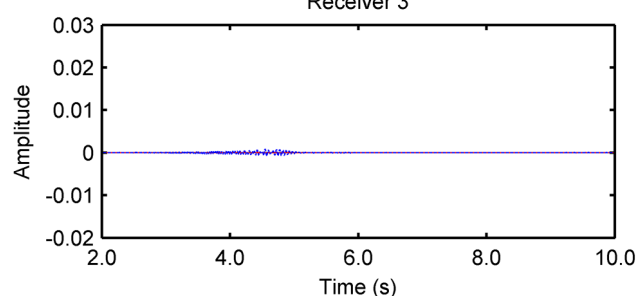

(d)

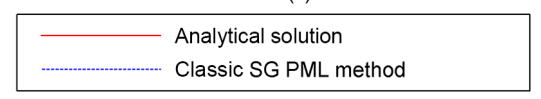

Receiver 1
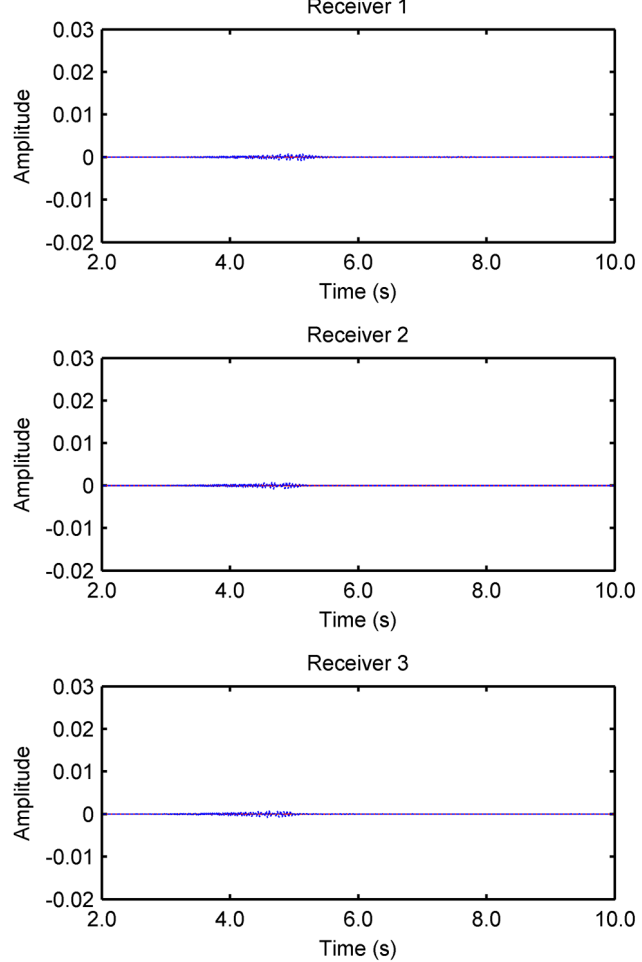

Figure 7. $d=10 \mathrm{~m}$; the rest is the same as in Fig. 6 . 
(a)
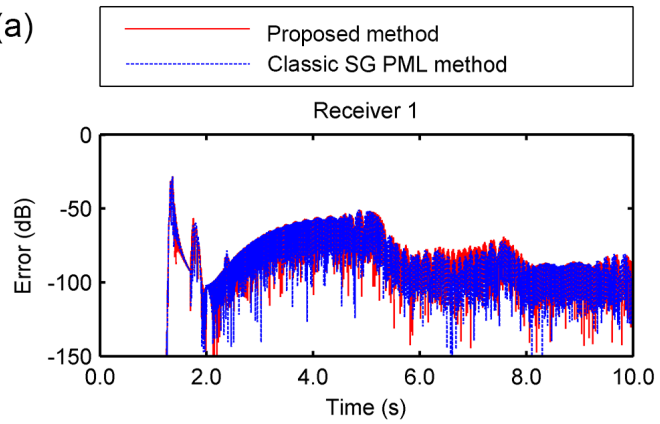

Receiver 2

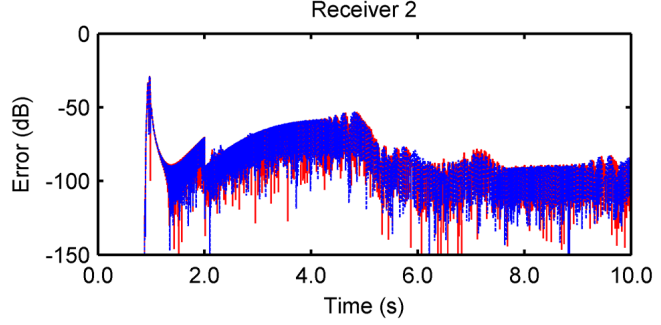

Receiver 3

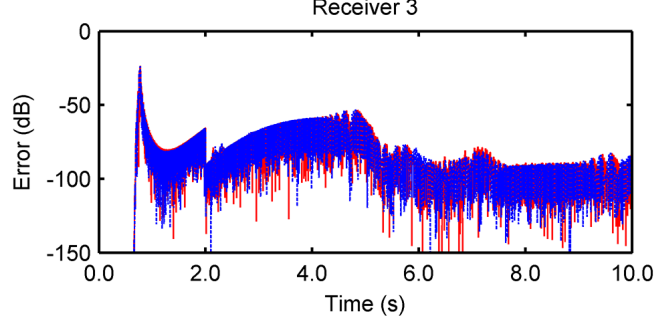

(b)
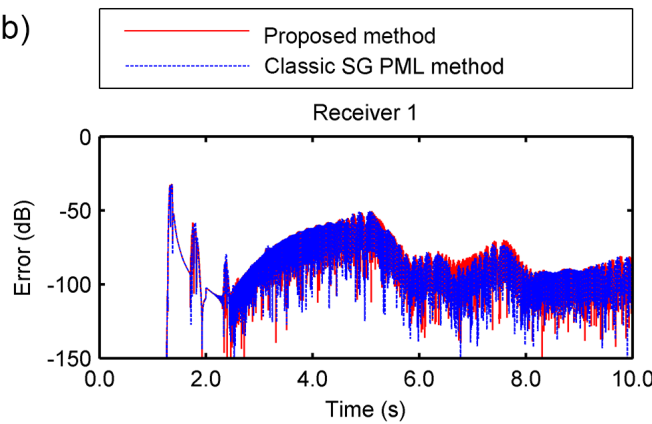

Receiver 2

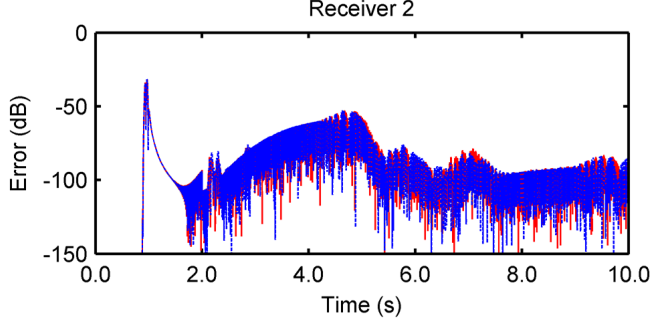

Receiver 3

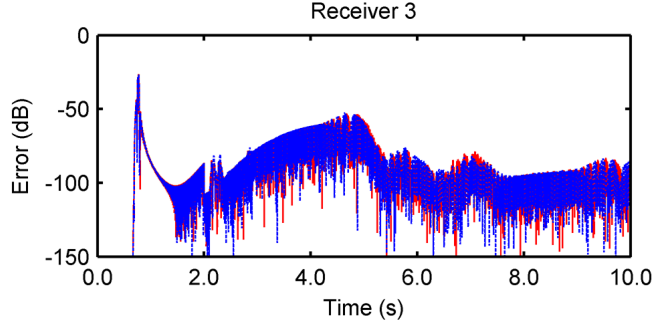

Figure 8. Comparison of the relative errors between the analytical solutions and the proposed method (fourth-order CG scheme) (red solid line) or the classic SG PML method (fourth-order SG scheme) (blue dotted line) at different receivers and different grid spacings. (a) $d=$ $12 \mathrm{~m}$, receivers 1,2 , and 3 ; (b) $d=10 \mathrm{~m}$, receivers 1,2 , and 3 .

lation for the acoustic wave equation in its standard secondorder form, while Pasalic and McGarry (2010) extended the convolutional PML to accommodate the second-order acoustic wave equation. Nevertheless, all of these second-order PML formulations require the derivation of complicated formulas, the introduction of auxiliary variables, and the modification of existing second-order numerical codes in order to handle the first-order equations describing the auxiliary variables, which increases the computational cost and complexity.

In order to preserve the original efficiency of the PML boundary processing method as well as the accuracy and efficiency of the CG scheme, it is worth trying to integrate the classic first-order PML algorithm into the CG finite-difference scheme in a second-order system and make it easy to implement. In this paper, we propose a new boundary-matched algorithm that uses a CG finite-difference scheme within a limited computational area and an SG finitedifference scheme in a PML area. Our approach enables the inner area and the PML condition to be independent during computation, while preserving the individual advantages of the two methods. The algorithm matches the computational area and the absorbing boundary layers simply by point updating along the boundaries of the computational area and avoids complex formula conversion. Thus, none of the original formulas of the CG scheme or the PML equations are modified and no unnecessary variables are added. The assessment of the proposed algorithm is composed of two parts. First, we compared the accuracy and efficiency of the proposed algorithm with those of the classic SG PML method (SG scheme both in computational area and PML area), which demonstrated the rationality of our decision to use the CG scheme in the computational area. To simulate the actual underground medium, a medium with a linearly increasing velocity gradient was selected for the experiment. The experimental results indicated that the accuracy of the two methods for equal grid sizes is almost equal, but the efficiency of our method is approximately $30 \%-50 \%$ higher than that of the classic SG PML method. Next, the proposed algorithm was evaluated by comparing its absorption efficiency and computational cost with those of the classic SG PML method, the second-order PML method (CG scheme both in computational area and PML area) introduced by Pasalic and McGarry (2010), and the hybrid ABC method (CG scheme 

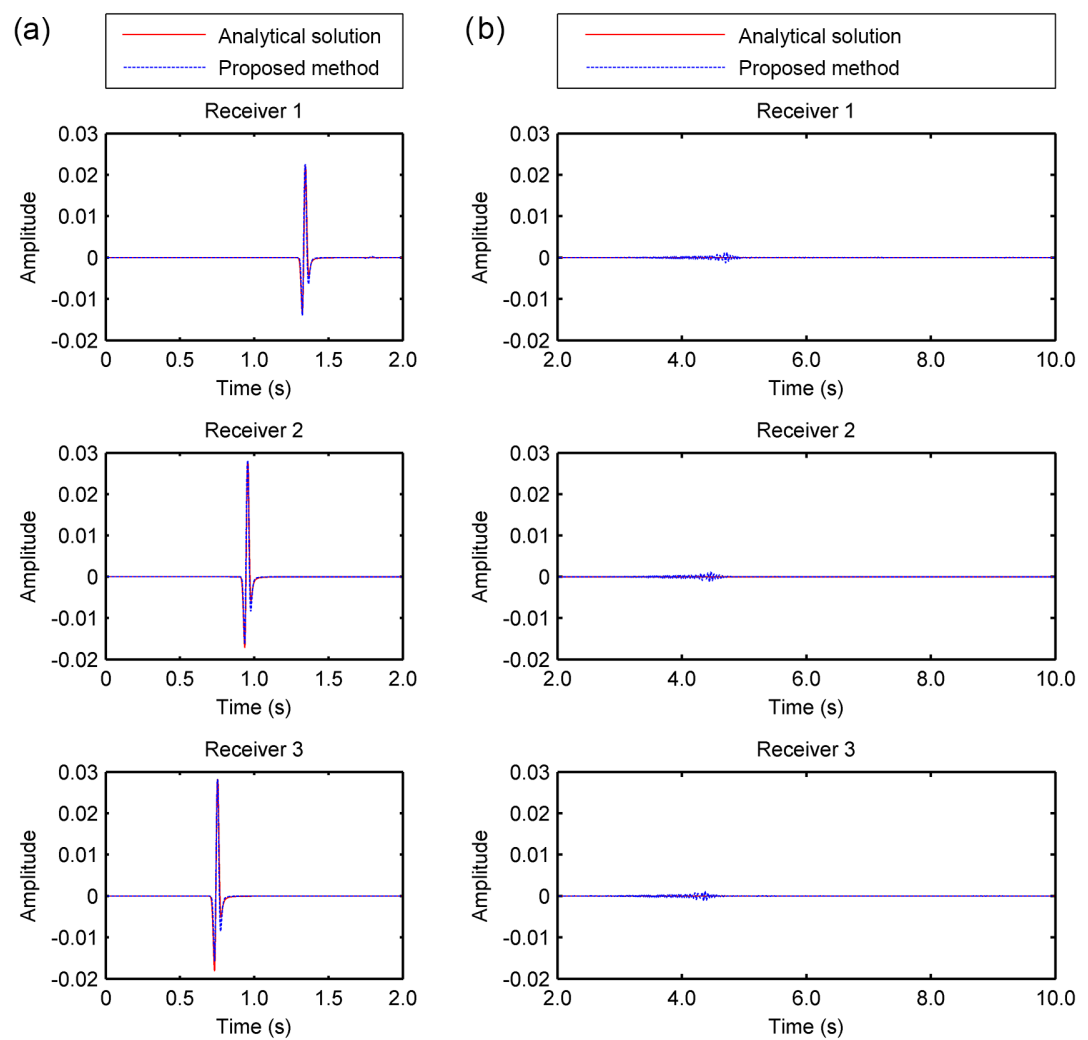

(c)

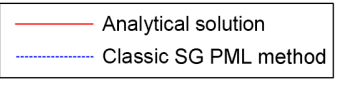

(d)
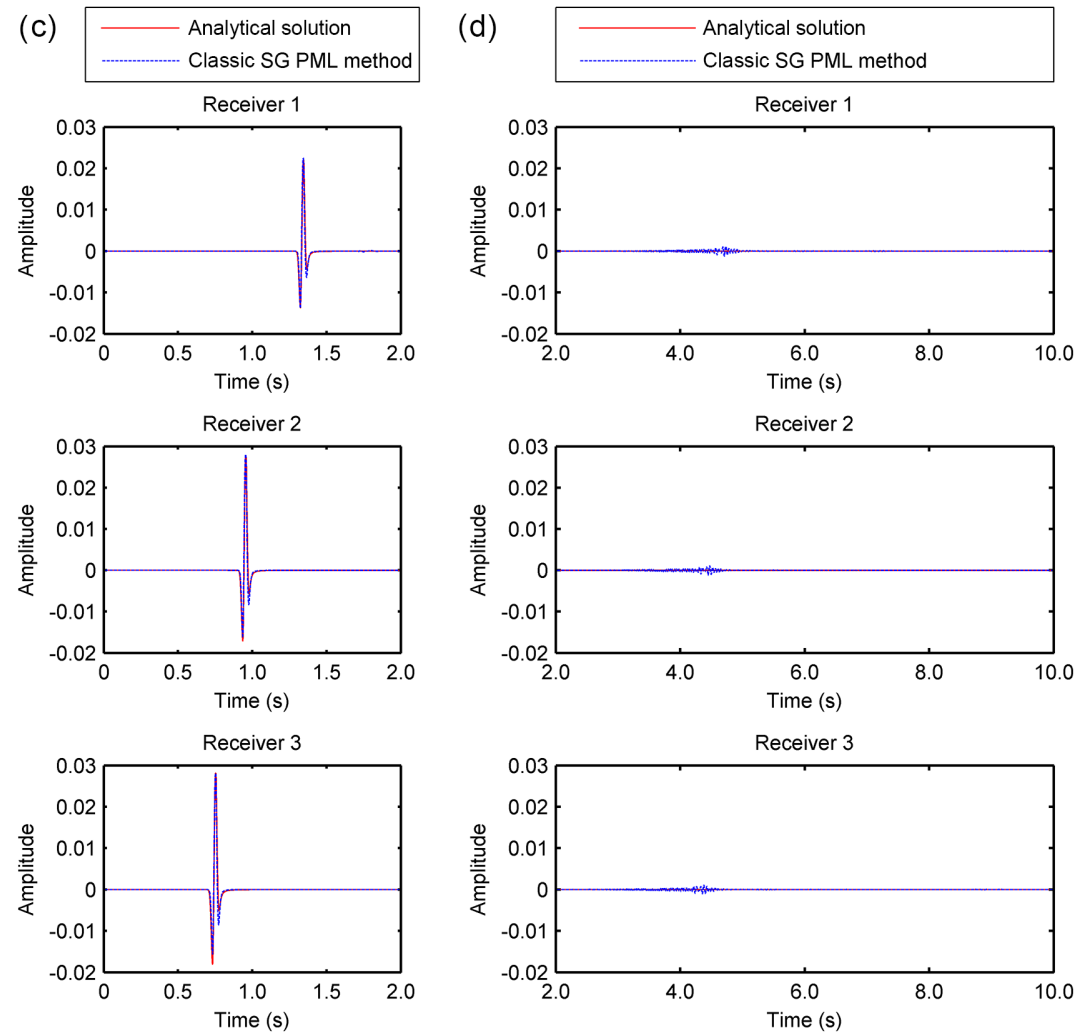

Figure 9. Comparison of the analytical solutions of the proposed (10th-order CG scheme) (red solid line) and the classic SG PML methods (10th-order SG scheme) (blue dotted line) at different receivers, $d=10 \mathrm{~m}$. (a) Proposed method at receivers 1, 2, and 3 during the first $2 \mathrm{~s}$; (b) proposed method at receivers 1, 2, and 3 after $2 \mathrm{~s}$; (c) classic SG PML method at receivers 1, 2, and 3 during the first $2 \mathrm{~s}$; (d) classic SG PML method at receivers 1,2 , and 3 after $2 \mathrm{~s}$. 

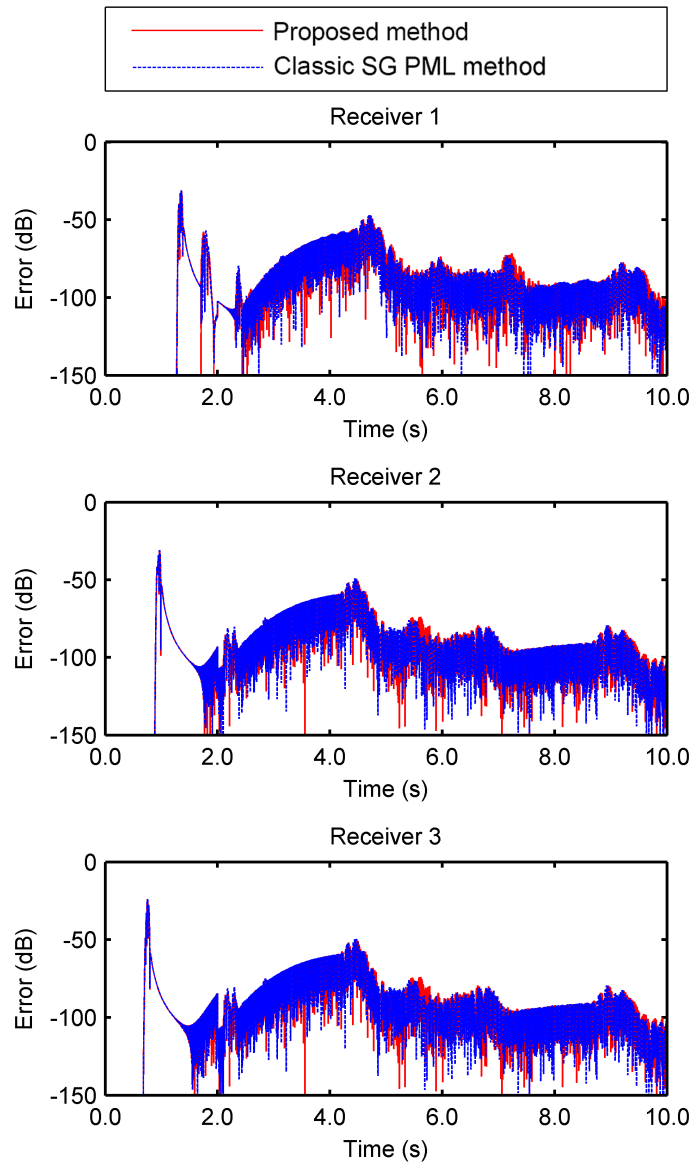

Figure 10. Comparison of the relative errors between the analytical solutions and the proposed method (10th-order CG scheme) (red solid line) or the classic SG PML method (10th-order SG scheme) (blue dotted line) at different receivers and different grid spacings $(d=10 \mathrm{~m}$, receivers 1,2 , and 3$)$.
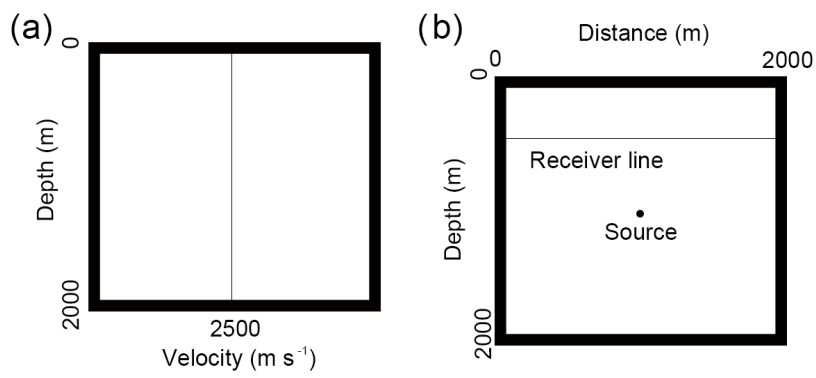

Figure 11. (a) Velocity profiles in depth; (b) distribution of source and receivers.

in computational area and hybrid $\mathrm{ABC}$ scheme in boundary area) introduced by Ren and Liu (2012). The numerical experimental results indicated that our algorithm provides an excellent absorption effect and was easier to implement.

\section{Methodology}

Although the elastic wave equation can describe the propagation of seismic waves more comprehensively, modeling an elastic wave field is complex and computationally expensive. In practice, the acoustic wave equation is also popularly used to approximate seismic wave propagation. For the convenient error analysis of these methods, we consider a scalar wave field $p$ propagating through an unbounded three-dimensional medium where the wave field satisfies Eq. (1) (Engquist and Runborg, 2003):

$\frac{\partial^{2} p}{\partial x^{2}}+\frac{\partial^{2} p}{\partial y^{2}}+\frac{\partial^{2} p}{\partial z^{2}}=\frac{1}{c^{2}} \cdot \frac{\partial^{2} p}{\partial t^{2}}$,

where the wave field $p$ is a function of the space variables $x, y, z$, and the time variable $t$, and $c$ is the velocity of the medium. Numeric modeling of Eq. (1) is expressed as follows.

\subsection{Conventional-grid finite-difference scheme}

The discretization of the acoustic wave (Eq. 1) with a $2 M$ order finite-difference scheme is (Chu and Stoffa, 2012)

$$
\begin{aligned}
p_{i, j, k}^{n+1} & =2 p_{i, j, k}^{n}-p_{i, j, k}^{n-1}+\frac{c^{2} \Delta t^{2}}{\Delta x^{2}}\left[c_{0} p_{i, j, k}^{n}\right. \\
& \left.+\sum_{m=1}^{M} c_{m}\left(p_{i-m, j, k}^{n}+p_{i+m, j, k}^{n}\right)\right] \\
& +\frac{c^{2} \Delta t^{2}}{\Delta y^{2}}\left[c_{0} p_{i, j, k}^{n}+\sum_{m=1}^{M} c_{m}\right. \\
& \left.\left(p_{i, j-m, k}^{n}+p_{i, j+m, k}^{n}\right)\right] \\
& +\frac{c^{2} \Delta t^{2}}{\Delta z^{2}}\left[c_{0} p_{i, j, k}^{n}+\sum_{m=1}^{M} c_{m}\right. \\
& \left.\left(p_{i, j, k-m}^{n}+p_{i, j, k+m}^{n}\right)\right],
\end{aligned}
$$

where $c_{m}$ for all $m$ are finite-difference coefficients. $i, j$, and $k$ denote the discrete spatial variables, and $n$ denotes the discrete time variable. The increments $\Delta x, \Delta y$, and $\Delta z$ are grid spacings, and $\Delta t$ is the time step. In many applications, a regular rectangular grid with a grid spacing $\Delta x=\Delta y=\Delta z=d$ is a natural and reasonable choice (Moczo et al., 2007).

Numerical analyses show that grid dispersion increases with increasing grid size, but decreasing the grid size increases the computational cost. High-order finite-difference schemes are able to control this numerical dispersion using a larger grid spacing compared with low-order schemes (Tan and Huang, 2014).

Because the subscripts $i, j$, and $k$ used in Eq. (2) have integer values, it is convenient to define and calculate the medium's parameters and wave field $p$ for the same grid points, which leads to the CG scheme. The pressure source $s$ is an additive item (Hustedt et al., 2004); i.e., it can be directly added in the corresponding equations. 

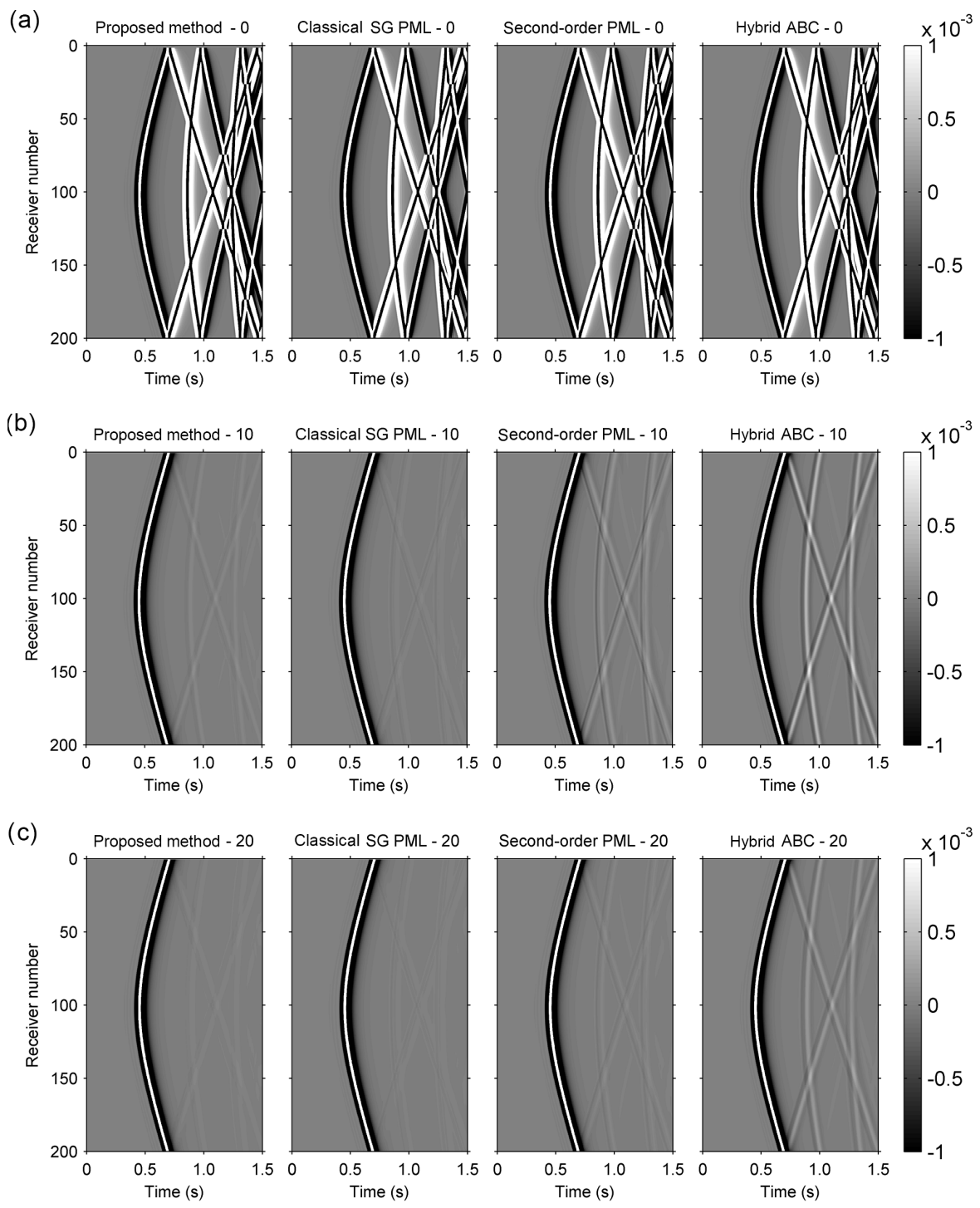

Figure 12. Receiver records of the four methods for a different number of absorbing layers: (a) 0 absorbing layers, (b) 10 absorbing layers, and (c) 20 absorbing layers.

\subsection{Boundary conditions}

Due to limitations in the capacity and speed of computer facilities, the numerical simulation of a wave field can only be implemented for a limited area. The computational area is surrounded by artificial boundaries, except for the free surface. As described above, the PML boundary condition can effectively absorb the wave field reflections from the artificial boundaries in order to simulate wave field propagation in an open space. In a PML medium, the wave field $p$ is assumed to be decomposed into subcomponents. The PML formulation based on the acoustic equations is as follows (Liu and Tao, 1998):

$\frac{\partial v_{x}}{\partial t}+\alpha_{x} v_{x}=-\frac{1}{\rho} \frac{\partial p}{\partial x}$

$$
\begin{aligned}
& \frac{\partial v_{y}}{\partial t}+\alpha_{y} v_{y}=-\frac{1}{\rho} \frac{\partial p}{\partial y}, \\
& \frac{\partial v_{z}}{\partial t}+\alpha_{z} v_{z}=-\frac{1}{\rho} \frac{\partial p}{\partial z}, \\
& \frac{\partial p_{x}}{\partial t}+\alpha_{x} p_{x}=-c^{2} \rho \frac{\partial v_{x}}{\partial x}, \\
& \frac{\partial p_{y}}{\partial t}+\alpha_{y} p_{y}=-c^{2} \rho \frac{\partial v_{y}}{\partial y}, \\
& \frac{\partial p_{z}}{\partial t}+\alpha_{z} p_{z}=-c^{2} \rho \frac{\partial v_{z}}{\partial z}, \\
& p=p_{x}+p_{y}+p_{z} .
\end{aligned}
$$

Here, $\alpha_{x}, \alpha_{y}$, and $\alpha_{z}$ are the attenuation coefficients in the PML medium. In this paper, the attenuation coefficient was 
(a)

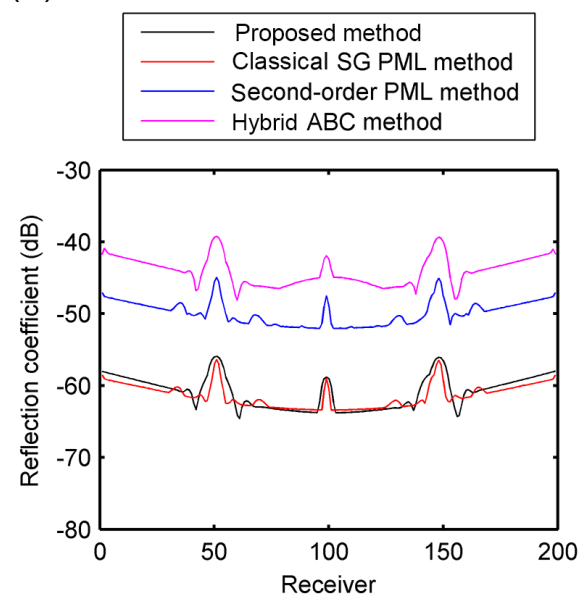

(b)

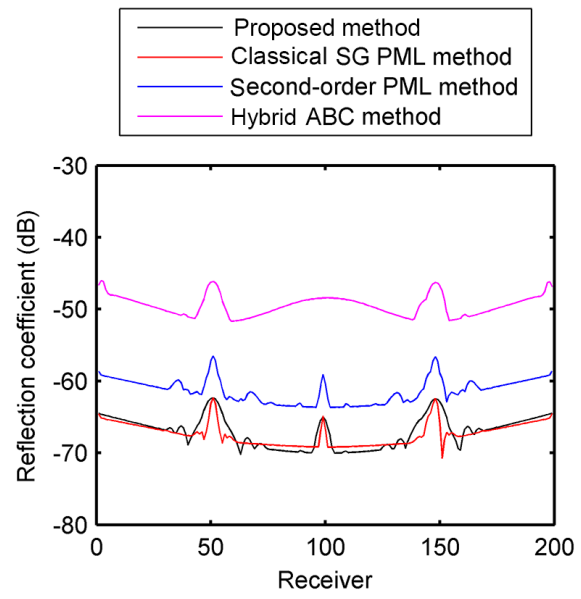

Figure 13. Values of the absorption coefficient $R$ at each receiver for our method (red line), the classic SG PML method (black line), the second-order PML method (blue line), and the hybrid ABC method (magenta line): (a) 10 absorbing layers and (b) 20 absorbing layers.
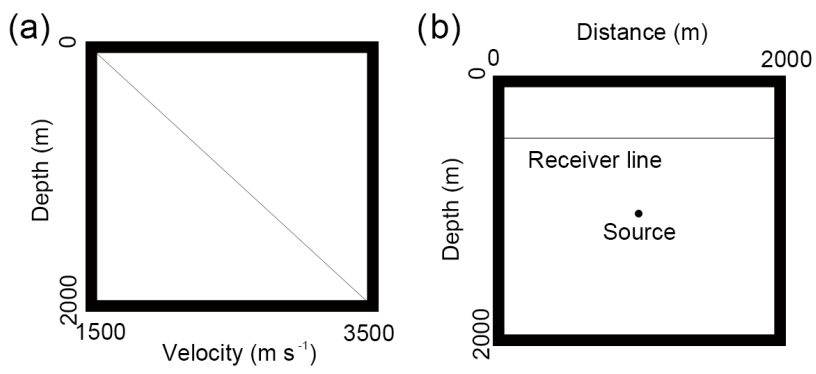

Figure 14. (a) Velocity profiles in depth; (b) distribution of source and receivers.

set using the following function (Wang, 2003):

$\alpha_{i j}=B\left[1-\sin \left(\frac{j \pi}{2 P_{\mathrm{ml}}}\right)\right], i=x y z ; j=0,1, \ldots, P_{\mathrm{ml}}$.

$B$ is the amplitude of attenuation coefficient, i.e., the maximum value of the coefficient, which we set as 400 in the numerical experiment; $P_{\mathrm{ml}}$ is the thickness of the PML layer.

Using the SG finite-difference scheme to discretize Eq. (3), the results are as follows:

$$
\begin{aligned}
& v_{x}^{n+\frac{1}{2}}\left(i+\frac{1}{2}, j, k\right)=v_{x}^{n-\frac{1}{2}}\left(i+\frac{1}{2}, j, k\right) \\
& \quad-\alpha_{x} \Delta t v_{x}^{n-\frac{1}{2}}\left(i+\frac{1}{2}, j, k\right) \\
& \quad-\frac{\Delta t}{\rho \Delta x}\left[p_{x}^{n}(i+1, j, k)+p_{y}^{n}(i+1, j, k)\right. \\
& \left.\quad+p_{z}^{n}(i+1, j, k)-p_{x}^{n}(i, j, k)-p_{y}^{n}(i, j, k)-p_{z}^{n}(i, j, k)\right], \\
& v_{y}^{n+\frac{1}{2}}\left(i, j+\frac{1}{2}, k\right)=v_{y}^{n-\frac{1}{2}}\left(i, j+\frac{1}{2}, k\right)
\end{aligned}
$$

$$
\begin{aligned}
& -\alpha_{y} \Delta t v_{y}^{n-\frac{1}{2}}\left(i, j+\frac{1}{2}, k\right)-\frac{\Delta t}{\rho \Delta y}\left[p_{x}^{n}(i, j+1, k)\right. \\
& +p_{y}^{n}(i, j+1, k)+p_{z}^{n}(i, j+1, k)-p_{x}^{n}(i, j, k) \\
& \left.-p_{y}^{n}(i, j, k)-p_{z}^{n}(i, j, k)\right], \\
& v_{z}^{n+\frac{1}{2}}\left(i, j, k+\frac{1}{2}\right)=v_{z}^{n-\frac{1}{2}}\left(i, j, k+\frac{1}{2}\right) \\
& -\alpha_{z} \Delta t v_{z}^{n-\frac{1}{2}}\left(i, j, k+\frac{1}{2}\right) \\
& -\frac{\Delta t}{\rho \Delta z}\left[p_{x}^{n}(i, j, k+1)+p_{y}^{n}(i, j, k+1)+p_{z}^{n}(i, j, k+1)\right. \\
& \left.-p_{x}^{n}(i, j, k)-p_{y}^{n}(i, j, k)-p_{z}^{n}(i, j, k)\right], \\
& p_{x}^{n+1}(i, j, k)=p_{x}^{n}(i, j, k)-\alpha_{x} \Delta t p_{x}^{n}(i, j, k) \\
& -\frac{c^{2} \rho \Delta t}{\Delta x}\left[v_{x}^{n+\frac{1}{2}}\left(i+\frac{1}{2}, j, k\right)-v_{x}^{n+\frac{1}{2}}\left(i-\frac{1}{2}, j, k\right)\right] \\
& p_{y}^{n+1}(i, j, k)=p_{y}^{n}(i, j, k)-\alpha_{y} \Delta t p_{y}^{n}(i, j, k) \\
& -\frac{c^{2} \rho \Delta t}{\Delta y}\left[v_{y}^{n+\frac{1}{2}}\left(i, j+\frac{1}{2}, k\right)-v_{y}^{n+\frac{1}{2}}\left(i, j-\frac{1}{2}, k\right)\right] \\
& p_{z}^{n+1}(i, j, k)=p_{z}^{n}(i, j, k)-\alpha_{z} \Delta t p_{z}^{n}(i, j, k) \\
& -\frac{c^{2} \rho \Delta t}{\Delta z}\left[v_{z}^{n+\frac{1}{2}}\left(i, j, k+\frac{1}{2}\right)-v_{z}^{n+\frac{1}{2}}\left(i, j, k-\frac{1}{2}\right)\right] .
\end{aligned}
$$

\subsection{Implementation of our new boundary-matched algorithm}

A finite-difference scheme based on a CG requires no computation of intermediate variables, and thus the computational cost is lower than that of an SG scheme. We will show in the next section that the accuracy of the CG scheme can reach the same level as that of the SG scheme but with lower computational costs. However, it is difficult to incorporate 

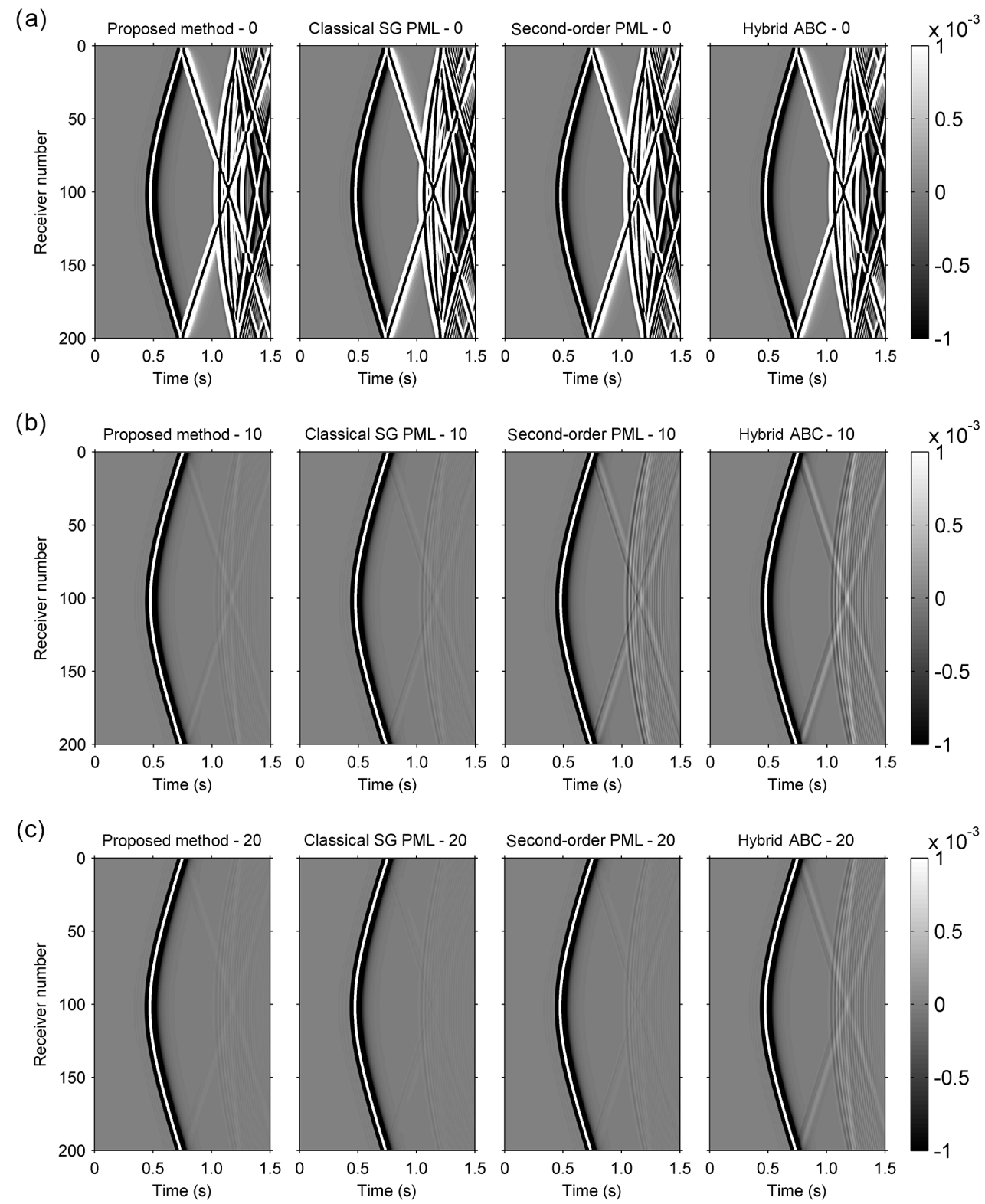

Figure 15. Receiver records for the four methods for a different number of absorbing layers: (a) 0 absorbing layers, (b) 10 absorbing layers, and (c) 20 absorbing layers.

a naturally formulated PML boundary processing algorithm based on an SG scheme into a CG finite-difference scheme. In this paper, we propose a new boundary-matched algorithm that can bridge the gap between an SG-based PML algorithm and a CG-based numerical simulation of a seismic wave field with neither introduction of intermediary variables nor reformulation of the PML equations. The core idea of the scheme is to interface the wave field reasonably along the boundaries between the CG area and the SG absorbing layers. A detailed description of the method is given below.

As shown in Fig. 1, the entire domain consists of two parts: the computational area and the boundary absorbing area. The computational area is located in the center and is surrounded by the absorbing layers. The algorithm uses a CG finitedifference scheme within the computational area and an SG finite-difference scheme within the boundary absorbing area. If we can reasonably interface the computed values of the wave field between the computational area and the boundary absorbing area, then the scheme can perform satisfactorily. For a clearer explanation, we start with a two-dimensional model.

We let the computational area and the PML area overlap each other for one layer. As shown in Fig. 1a, the bold red boundary line is both the outermost boundary of the compu- 
(a)

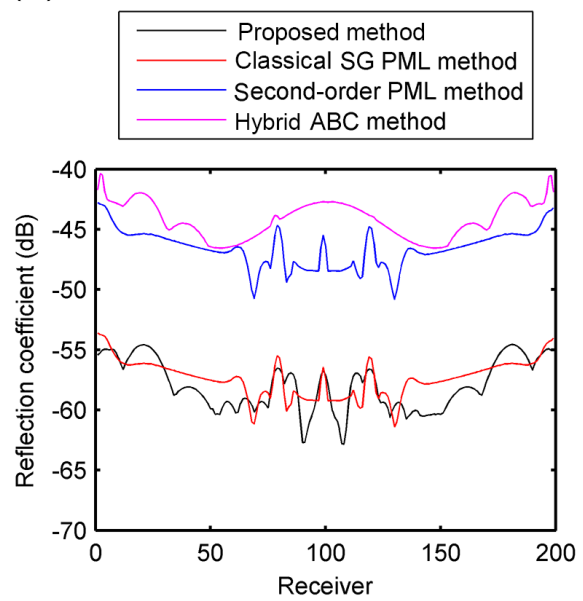

(b)

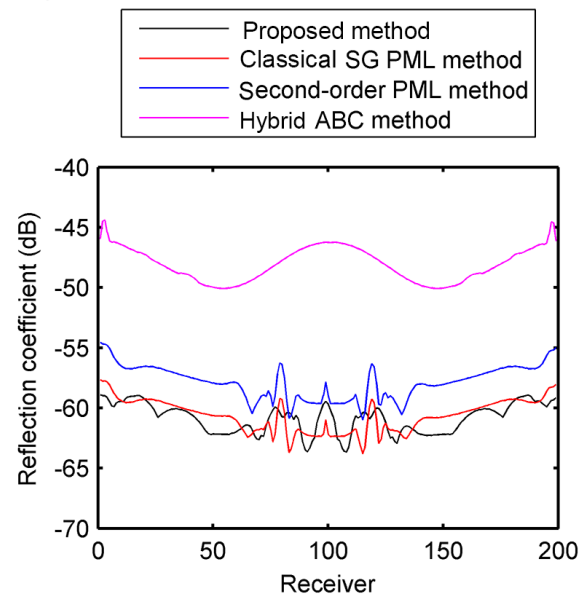

Figure 16. Values of the absorption coefficient $R$ at each receiver for our method (red line), the classic SG PML method (black line), the second-order PML method (blue line), and the hybrid ABC method (magenta line): (a) 10 absorbing layers and (b) 20 absorbing layers.

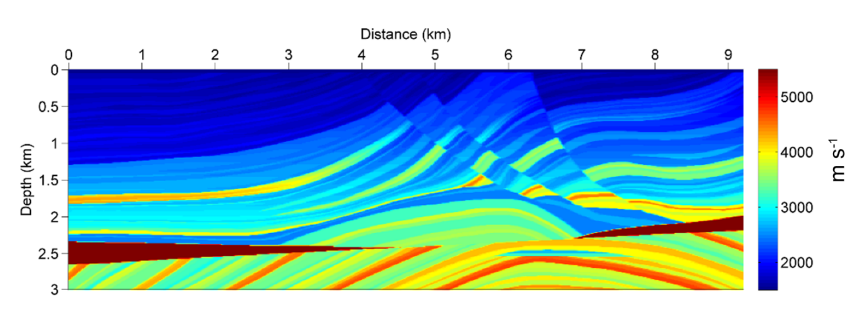

Figure 17. Marmousi velocity model.

tational area and the innermost boundary of the PML area. On this overlapped layer, both the particle velocity $v$ and the wave field $p$ in the PML area are calculated using the value of wave field $p$ in the computational area. Using this method, the two areas can be connected. This avoids the introduction of intermediate variables and saves storage space. In order to distinguish, we use $p_{i, j}^{n+1}$ to represent the wave field value in the computational area, and $p_{x}^{n}(i j)$ and $p_{z}^{n}(i j)$ to represent the wave field value in the PML area. In the PML area, the values of attenuation coefficients $\alpha_{x}$ and $\alpha_{z}$ can be calculated using Eq. (4). When the grid points are located on the four corners of the PML area, the values of $\alpha_{x}$ and $\alpha_{z}$ are not zero. When they are on the left- and right-hand sides of the PML area, $\alpha_{x} \neq 0$ and $\alpha_{z}=0$; when they are on the upper and bottom sides of the PML area, $\alpha_{x}=0$ and $\alpha_{z} \neq 0$. The specific steps of our method are as follows.

1. At the beginning of iteration, take $n=1$, and let the initial wave field values $p_{i, j}^{n}$ and $p_{i, j}^{n-1}$ in the computational area, the particle velocity $v_{x}^{n-\frac{1}{2}}\left(i+\frac{1}{2}, j\right)$ and $v_{z}^{n-\frac{1}{2}}\left(i, j+\frac{1}{2}\right)$, the wave field $p_{x}^{n}(i j)$ and $p_{z}^{n}(i j)$ in the PML area all be zero.
2. Calculate the wave field $p_{i, j}^{n+1}$ in the computational area. In this step, we do not calculate the value of wave field $p_{i, j}^{n+1}$ located on the red boundary line; instead, we only calculate them on the blue boundary line and in its inner region in Fig. 1 using the two-dimensional form of Eq. (2). For the high-order difference scheme in the computational area, we use the second-order difference scheme for the grid points on the blue rectangular line, the fourth-order difference scheme for the grid points on the inner green rectangular line, the sixth-order difference scheme on the inner layer, and so on, until we reach the required order of difference. In this way, the computational area and the PML area can be independent, and the number of overlapping layers does not increase with the increase of order of difference. The numerical experiments in Sect. 4.1 will prove that our treatment to the boundary does not bring much additional dispersion and error.

3. Calculate the particle velocity and wave field in the PML area layer by layer. Calculate the values of all particle velocity $v_{x}^{n+\frac{1}{2}}\left(i+\frac{1}{2}, j\right)$ and $v_{z}^{n+\frac{1}{2}}\left(i, j+\frac{1}{2}\right)$ in the PML area (including those on the red line) using the two-dimensional forms of the first and third formulas in Eq. (5). Calculate all of the wave field values $p_{x}^{n+1}(i j)$ and $p_{z}^{n+1}(i j)$ in the PML area (including those on the red line) using the two-dimensional forms of the fourth and sixth formulas in Eq. (5).

It is important to note that the particle velocities in the area between the blue and red lines are calculated from the wave field $p_{x}^{n}\left(i_{\mathrm{r}} j_{\mathrm{r}}\right)$ and $p_{z}^{n}\left(i_{\mathrm{r}} j_{\mathrm{r}}\right)$ on the red line in the PML area and the wave field $p_{i_{\mathrm{b}}, j_{\mathrm{b}}}^{n}$ on the blue line in the computational area. As shown in Fig. 1b, we can 

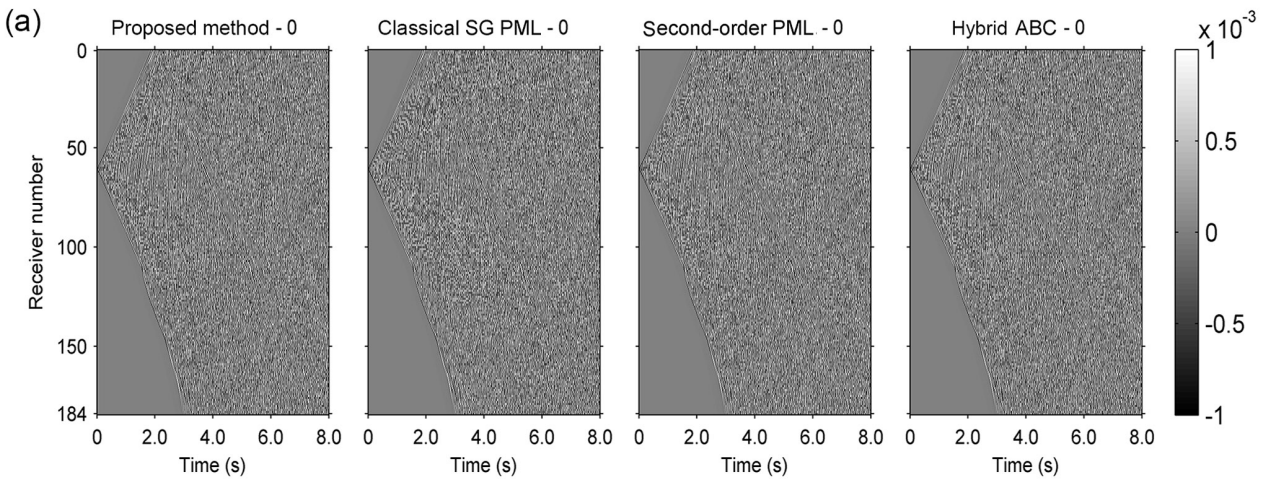

(b)
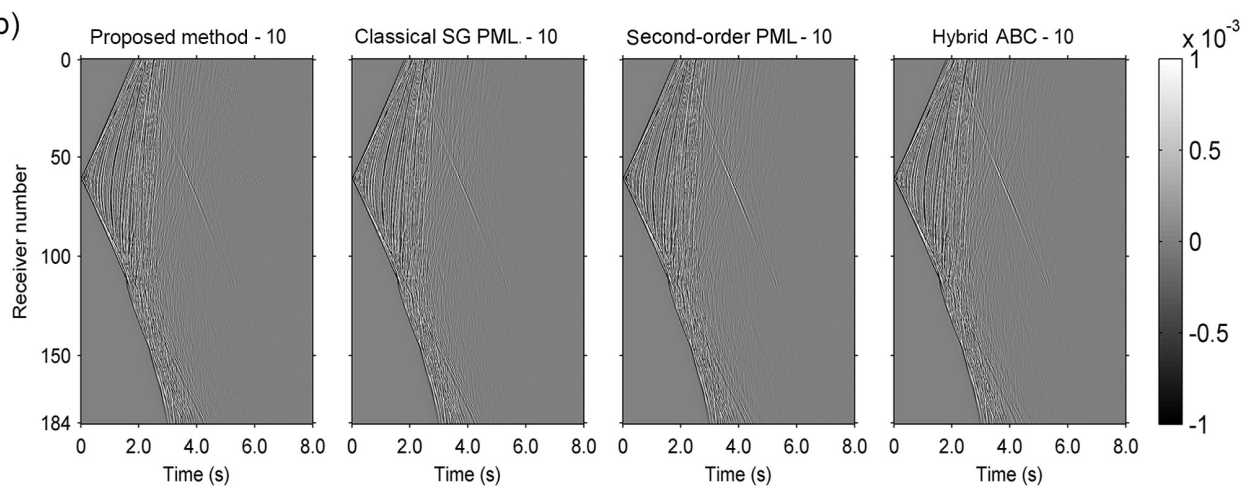

(c)
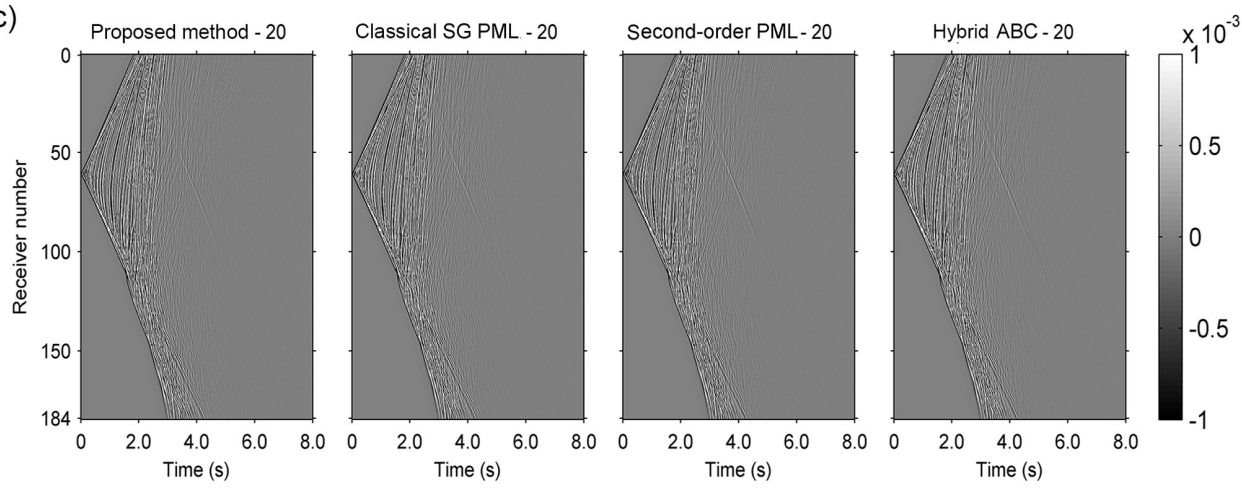

Figure 18. Receiver records for the four methods for a different number of absorbing layers: (a) 0 absorbing layers, (b) 10 absorbing layers, and (c) 20 absorbing layers.

obtain $v_{x}^{n+\frac{1}{2}}\left(i_{\mathrm{r}}+\frac{1}{2}, j_{\mathrm{r}}\right)$ for the line between the lefthand line of the red rectangle and blue rectangle using Eq. (6); the subscript $r$ stands for the grid points on the red lines, and $b$ stands for the blue lines. Similarly, we can obtain $v_{x}^{n+\frac{1}{2}}\left(i_{\mathrm{r}}-\frac{1}{2}, j_{\mathrm{r}}\right)$ between the righthand line of two rectangles using Eq. (7); for the line between the upper lines, we obtain $v_{z}^{n+\frac{1}{2}}\left(i_{\mathrm{r}}, j_{\mathrm{r}}+\frac{1}{2}\right)$ using Eq. (8); for the line between the lower lines, we obtain $v_{z}^{n+\frac{1}{2}}\left(i_{\mathrm{r}}, j_{\mathrm{r}}-\frac{1}{2}\right)$ using Eq. (9):

$$
v_{x}^{n+\frac{1}{2}}\left(i_{\mathrm{r}}+\frac{1}{2}, j_{\mathrm{r}}\right)=v_{x}^{n-\frac{1}{2}}\left(i_{\mathrm{r}}+\frac{1}{2}, j_{\mathrm{r}}\right)
$$

$$
\begin{aligned}
& -\alpha_{x} \Delta t v_{x}^{n-\frac{1}{2}}\left(i_{\mathrm{r}}+\frac{1}{2}, j_{\mathrm{r}}\right) \\
& -\frac{\Delta t}{\rho \Delta x}\left[p_{i_{\mathrm{b}}, j_{\mathrm{b}}}^{n}-p_{x}^{n}\left(i_{\mathrm{r}}, j_{\mathrm{r}}\right)-p_{z}^{n}\left(i_{\mathrm{r}}, j_{\mathrm{r}}\right)\right], \\
& v_{x}^{n+\frac{1}{2}}\left(i_{\mathrm{r}}-\frac{1}{2}, j_{\mathrm{r}}\right)=v_{x}^{n-\frac{1}{2}}\left(i_{\mathrm{r}}-\frac{1}{2}, j_{\mathrm{r}}\right) \\
& -\alpha_{x} \Delta t v_{x}^{n-\frac{1}{2}}\left(i_{\mathrm{r}}-\frac{1}{2}, j_{\mathrm{r}}\right) \\
& -\frac{\Delta t}{\rho \Delta x}\left[p_{x}^{n}\left(i_{\mathrm{r}}, j_{\mathrm{r}}\right)+p_{z}^{n}\left(i_{\mathrm{r}}, j_{\mathrm{r}}\right)-p_{i_{\mathrm{b}}, j_{\mathrm{b}}}^{n}\right], \\
& v_{z}^{n+\frac{1}{2}}\left(i_{\mathrm{r}}, j_{\mathrm{r}}+\frac{1}{2}\right)=v_{z}^{n-\frac{1}{2}}\left(i_{\mathrm{r}}, j_{\mathrm{r}}+\frac{1}{2}\right)
\end{aligned}
$$



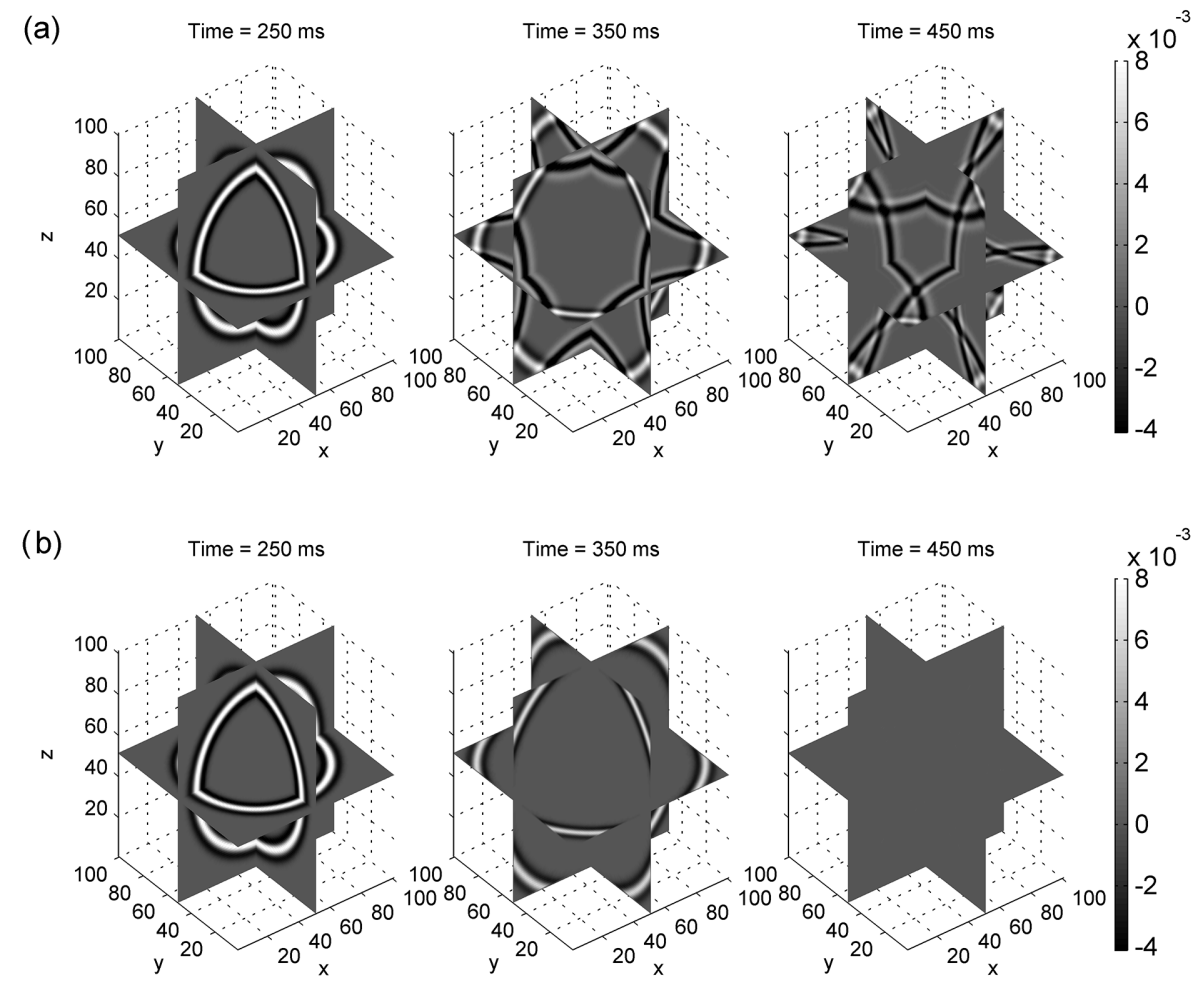

Figure 19. Wave field snapshots with different PML at different time; the three coordinate axes represent the number of grids in three directions: (a) PML is 0 at 250, 350, and $450 \mathrm{~ms}$; (b) PML is 20 at 250, 350, and $450 \mathrm{~ms}$.

$$
\begin{aligned}
& -\alpha_{z} \Delta t v_{z}^{n-\frac{1}{2}}\left(i_{\mathrm{r}}, j_{\mathrm{r}}+\frac{1}{2}\right) \\
& -\frac{\Delta t}{\rho \Delta z}\left[p_{i_{\mathrm{b}}, j_{\mathrm{b}}}^{n}-p_{x}^{n}\left(i_{\mathrm{r}}, j_{\mathrm{r}}\right)-p_{z}^{n}\left(i_{\mathrm{r}}, j_{\mathrm{r}}\right)\right], \\
& v_{z}^{n+\frac{1}{2}}\left(i_{\mathrm{r}}, j_{\mathrm{r}}-\frac{1}{2}\right)=v_{z}^{n-\frac{1}{2}}\left(i_{\mathrm{r}}, j_{\mathrm{r}}-\frac{1}{2}\right) \\
& -\alpha_{z} \Delta t v_{z}^{n-\frac{1}{2}}\left(i_{\mathrm{r}}, j_{\mathrm{r}}-\frac{1}{2}\right) \\
& -\frac{\Delta t}{\rho \Delta z}\left[p_{x}^{n}\left(i_{\mathrm{r}}, j_{\mathrm{r}}\right)+p_{z}^{n}\left(i_{\mathrm{r}}, j_{\mathrm{r}}\right)-p_{i_{\mathrm{b}}, j_{\mathrm{b}}}^{n}\right] .
\end{aligned}
$$

After calculating the complete PML area, let the value of the wave field $p_{i, j}^{n+1}$ on the red line in the computational area be equal to the sum of the wave field $p_{x}^{n+1}\left(i_{\mathrm{r}} j_{\mathrm{r}}\right)$ and $p_{z}^{n+1}\left(i_{\mathrm{r}} j_{\mathrm{r}}\right)$ on the red line in the PML area.

4. Update the value of $p_{i, j}^{n-1}$ with the value of $p_{i, j}^{n}$, and update the value of $p_{i, j}^{n}$ with the value of $p_{i, j}^{n+1}$; then, let $n=n+1$.

5. Repeat steps $2-4$ until $n$ reaches the required time length.

The two-dimensional algorithm described above can easily be generalized to three-dimensional. In the threedimensional model, we need to add a particle velocity com- ponent $v_{y}$ and a space position label $k$. The red and blue boundary lines become the red and blue boundary surfaces, respectively. In addition, the computational area becomes a cube surrounded by the PML area.

\section{Performance analysis}

As described in the introduction, the errors in the wave field numerical model are mainly caused by differential dispersion and reflected waves that are not fully absorbed by the boundary processing algorithm. In order to verify the validity of our algorithm, we used a variety of models to compare the computational accuracy, the efficiency of the absorption of the reflected waves, and the computational efficiency of the proposed algorithm to the other methods.

\subsection{Computational accuracy}

In order to obtain a more convincing result when comparing the computational accuracy, we used a constant-gradient velocity model, the velocity of which increases linearly with depth. This model is closer to the actual velocity distribution of an underground medium than a homogeneous model. We calculated the relative error between our method and the classic SG PML method using the analytical solutions for different grid spacings and the order of difference, and then 
we performed a comparative analysis of the two methods. The relative error between the two methods and the analytical solution is defined by the following time function:

$\operatorname{error}(t)=20 \log \left|\frac{p(t)-p_{\text {anal }}(t)}{\max \left[p_{\text {anal }}(t)\right]}\right|$.

In Eq. (10), $p(t)$ represents the value of wave field calculated by the numerical methods at a receiver, and $p_{\text {anal }}(t)$ is the value of wave field calculated by the analytic solution at the same receiver.

For the two-dimensional scalar (Eq. 1), the wave field analytic solution for a constant-gradient medium can be obtained from the integral form of the three-dimensional solution using the dimension reduction method (Cerveny, 2001). The velocity distribution is $c(z)=c\left(z_{0}\right) \frac{1+\gamma z}{1+\gamma z 0}=c(0)(1+\gamma z)$, where $z_{0}$ is the depth of source, $c\left(z_{0}\right)$ is the velocity of the source layer, $c(0)$ is the velocity of the layer $z=0$, and $\gamma=\frac{1}{h}, h$ is the distance from depth $z=0$ to the level where the propagation velocity is null $(c(-h)=0)$. When the line source $s=\delta\left(t-t_{0}\right) \delta\left(x-x_{0}\right) \delta\left(z-z_{0}\right)$ is located at $\left(x_{0} z_{0}\right)$, and the density in the method of Sanchez-Sesma et al. (2001) is a constant, a two-dimensional scalar Green function can be obtained by

$G(x z t) \approx \Lambda \cdot \frac{H\left(t-t_{0}-\tau\right)}{2 \pi \sqrt{\left(t-t_{0}\right)^{2}-\tau^{2}}}$,

where $\Lambda=\sqrt{\frac{1+\gamma z_{0}}{1+\gamma z} \cdot \frac{c\left(z_{0}\right) \tau}{R_{\mathrm{w}}}}$.

$H(t)$ is the Heaviside step function (equal to 0 when $t<0$ and equal to 1 when $t>0$ ). For fixed $t$, the radius $R_{\mathrm{w}}$ of the wavefront circle is $R_{\mathrm{w}}=\left(z_{0}+h\right) \sin h(\gamma c(0) \tau)$ and the travel time $\tau$ can be computed by means (Cerveny, 2001) of $\tau=\left|\frac{1}{\gamma c(0)} \operatorname{arccosh}\left[1+\frac{(\gamma c(0) r)^{2}}{2 c(z) c\left(z_{0}\right)}\right]\right|, r=$ $\sqrt{\left(x-x_{0}\right)^{2}+\left(z-z_{0}\right)^{2}}$. This is an approximate solution, but usually the error is less than $1 \%$ (Sanchez-Sesma et al., 2001). In the next numerical experiment, we use a symmetric Ricker wavelet with a peak frequency of $20 \mathrm{~Hz}$ as the source. In this paper, the expression is usually $s(t)=$ $\left\{1-2\left[20 \pi\left(t-t_{0}-1 / 20\right)\right]^{2}\right\} e^{-}\left[20 \pi\left(t-t_{0}-1 / 20\right)\right]^{2}$, and $t_{0}$ is equal to $200 \mathrm{~ms}$. The final result of the analytic solution is

$p_{\text {anal }}(x, z, t)=G(x, z, t) \times \delta\left(x-x_{0}\right) \delta\left(z-z_{0}\right) S(t)$.

\subsection{Absorption efficiency of the reflected waves}

When comparing the absorption efficiency, we used three different geological models to determine the reflected wave absorption effect of our algorithm: the homogeneous, constantgradient velocity and the Marmousi models. We compared the absorption effects of our algorithm with the classic SG PML method, the second-order PML method, and the hybrid $\mathrm{ABC}$ method using the same conditions to prove whether our algorithm can effectively combine the CG scheme with the SG scheme PML boundary condition and achieve the same or better effect as other methods do. In the computational area, the reflection coefficient $R$ of a receiver is defined as

$R=20 \log \left|\frac{\max \left[p(t)-p_{\text {ref }}(t)\right]}{\max \left[p_{\text {ref }}(t)\right]}\right|$,

where the wave field value $p(t)$ is calculated by the numerical methods at a receiver, and $p_{\text {ref }}(t)$ is the wave field value that has no boundary reflection on the same receiver calculated using the numerical methods, which can be obtained by expanding the model. The value of $R$ reflects the reflected wave absorption effect of the algorithm. The smaller the $R$ value, the better the absorption effect.

\subsection{Computational efficiency index}

In the comparison of the computational accuracy and efficiency of the absorption of the reflected waves, we determined the computation time of the three methods separately, which can reflect the advantages and disadvantages of all of the methods in terms of the computational efficiency.

\section{Numerical experiment}

Based on the discussion of the performance analysis, in this section, we present the results of the numerical experiments. All of the numerical experiments were run on a desktop personal computer with a $3.40 \mathrm{GHz}$ Intel Core i5-3570 processor, 32 GB of DDR3 memory, on a 64 bit Windows 7 operating system, using algorithmic software written in $\mathrm{C}++$.

\subsection{Computational accuracy and computation time}

As shown in Fig. 2, the constant-gradient velocity model has a size of $6000 \mathrm{~m} \times 6000 \mathrm{~m}$, a velocity distribution of $c=$ $500 \mathrm{~m} \mathrm{~s}^{-1}$ for $z=0 \mathrm{~m}$ and $c=5300 \mathrm{~m} \mathrm{~s}^{-1}$ for $z=6000 \mathrm{~m}$, and a velocity gradient of 0.8 . In order to determine the stability of the differential form throughout the computational process, the time step was set as $0.001 \mathrm{~s}$ and the total simulation time as $10 \mathrm{~s}$. The sources are located in the middle of the model $(3000,3000 \mathrm{~m})$, and the three receivers are located at $(600,1800 \mathrm{~m}),(1800,1800 \mathrm{~m})$, and $(3000,1800 \mathrm{~m})$. We compared the errors of the numerical solution and the analytical solution at the receivers of the method we proposed with the classic SG PML methods. The number of PMLs is set as 10 . Figures 3 and 4 show the comparison of the analytical solutions at the three receivers of the proposed method (secondorder CG scheme in the computational area) and the classic SG PML method (second-order SG scheme in the computational area) when the grid spacing $(d)$ is 12 and $10 \mathrm{~m}$, respectively. Figure 5 shows the relative errors between the analytical solutions and the two methods for the conditions described above where the relative error was calculated using Eq. (10).

From Figs. 3 and 4, we can see that both of the methods have obvious errors during the first $2 \mathrm{~s}$. In particular, when 
the grid spacing is $12 \mathrm{~m}$, the error is the largest, and there is significant numerical dispersion. Reducing the grid spacing can reduce the error and the dispersion. When the grid spacing is $10 \mathrm{~m}$, the result improves. In addition, the results for a longer simulation time also prove the numerical stability of our method. Further comparison of the relative error curves shown in Fig. 5 indicates that although neither method is particularly good; the amplitude of relative errors with their analytical solutions are almost the same.

In theory, the error of the numerical solution can be reduced by using a higher-order difference. We compared the experimental results of the proposed method (fourth-order CG scheme in the computational area) and classic SG PML methods (fourth-order SG scheme in the computational area) with the analytic solution, as shown in Figs. 6-8.

From Figs. 6 and 7, we can see that when the fourth-order difference is used, the relative errors between the analytical solution and both methods are significantly reduced compared with when the second-order difference is used. In addition, as with the second-order result above, the relative error also decreases as the grid spacing decreases. Figure 8 illustrates the fact that the relative error curves of our algorithm and the classic SG PML method are also very similar for the fourth-order difference, In addition, it is difficult to distinguish the advantages and disadvantages of the two algorithms. Although the results of the two methods still exhibit a small error at this time, we can continue to use the higher difference order or we can reduce the grid spacing to reduce the error. The laws of the two methods are the same.

In Figs. 9 and 10, we adopt a 10th-order difference scheme and $d=10 \mathrm{~m}$. At this time, the numerical results are very close to the analytical solution and the relative error is very low. Based on this, we can conclude that the accuracies of the proposed method and the classic SG PML method for the same conditions with a constant-gradient velocity are similar. Meanwhile, it also demonstrates an additional advantage of our method. When the computational area and the PML area are independent of each other, we can easily optimize the numerical algorithm of the computational area to improve the accuracy of the algorithm. When the grid spacing and the order of difference are appropriate, our method yields the expected results. Because our method uses the CG scheme in the computational area, the experimental results also show that in the scalar wave field simulation, the accuracy of the SG scheme is not higher than that of the CG scheme. This conclusion is in agreement with the experimental results of the elastic wave field simulated by Moczo et al. (2011) at a low $P$-wave to $S$-wave speed ratio $\left(V_{p} / V_{s}=1.42\right)$.

Table 1 presents the computation times of the two methods at different grid spacings and difference orders. The efficiency percentage is the total computation time of our method divided by the total computation time of the SG method. Under the same circumstances, the total computation time of our method is only $57 \%-70 \%$ that of the classic SG PML method. It is noteworthy that the result of our
Table 1. Computation time for our and the classic SG PML methods.

\begin{tabular}{llll}
\hline Condition & Our method & $\begin{array}{l}\text { The classic } \\
\text { SG PML method }\end{array}$ & $\begin{array}{l}\text { Efficiency } \\
\text { percentage }\end{array}$ \\
\hline $\begin{array}{l}\text { Second-order } \\
d=12 \mathrm{~m}\end{array}$ & $10 \mathrm{~m} 19 \mathrm{~s}$ & $15 \mathrm{~m} 17 \mathrm{~s}$ & $67.5 \%$ \\
$\begin{array}{l}\text { Second-order } \\
d=10 \mathrm{~m}\end{array}$ & $14 \mathrm{~m} 41 \mathrm{~s}$ & $21 \mathrm{~m} 01 \mathrm{~s}$ & $69.8 \%$ \\
$\begin{array}{l}\text { Fourth-order } \\
d=12 \mathrm{~m}\end{array}$ & $11 \mathrm{~m} 13 \mathrm{~s}$ & $17 \mathrm{~m} 56 \mathrm{~s}$ & $62.5 \%$ \\
$\begin{array}{l}\text { Fourth-order } \\
d=10 \mathrm{~m}\end{array}$ & $16 \mathrm{~m} 58 \mathrm{~s}$ & $25 \mathrm{~m} 54 \mathrm{~s}$ & $64.3 \%$ \\
$\begin{array}{l}10 \text { th-order } \\
d=10 \mathrm{~m}\end{array}$ & $25 \mathrm{~m} 48 \mathrm{~s}$ & $44 \mathrm{~m} 54 \mathrm{~s}$ & $57.4 \%$ \\
\hline
\end{tabular}

method for the 10th-order difference and a grid spacing of $10 \mathrm{~m}$ is much better than that of the classic SG PML method for the fourth-order difference and a $10 \mathrm{~m}$ grid spacing, while the computation time is nearly the same. Also, the result of our method for the fourth-order difference and a grid spacing of $12 \mathrm{~m}$ is much better than that of the classic SG PML method for the second-order difference and a $10 \mathrm{~m}$ grid spacing, while the former computation time is only $53.3 \%$ of the latter. Therefore, for the same computation time as the classic SG PML method, our method always achieves a higher accuracy for a smaller grid spacing and a higher-order difference. We obtained these conclusions in a constant-gradient velocity medium. Therefore, the algorithm we propose works well when the CG scheme is used in the computational area. Next, we discuss the absorption efficiency of the reflected waves of our method in a series of simple and complex models.

\subsection{Absorption efficiency and computation time}

\subsubsection{Homogeneous model}

First, we used a two-dimensional homogeneous model to verify the reflected wave absorption efficiency of our new boundary-matched algorithm. As shown in Fig. 11, the model size is $2000 \mathrm{~m} \times 2000 \mathrm{~m}$ at a velocity of $c=$ $2500 \mathrm{~m} \mathrm{~s}^{-1}$ and a grid spacing of $10 \mathrm{~m}$. The source is the same as previously described and is located at $(1000$, $1000 \mathrm{~m}$ ) with a time step of $0.001 \mathrm{~s}$ and a total simulation time of $1.5 \mathrm{~s}$. A total of 201 receivers are evenly distributed on a horizontal line with a depth of $500 \mathrm{~m}$, and the distance between each receiver is set as $10 \mathrm{~m}$. In Fig. 12, we compared the receiver records of our method, the classic SG PML method, the second-order PML method, and the hybrid ABC method for a different number of absorbing layers. In general, the amplitude of the reflected wave will be reduced to less than $1 \%$ of that of the normal wave field after the boundary conditions are processed. Thus, in order to illustrate the reflected wave more clearly, we set the range of the color bar of the wave field to be -0.001 to 0.001 . For further com- 
Table 2. Computation times for the four methods.

\begin{tabular}{|c|c|c|c|c|}
\hline Condition & Our method & Classic SG PML method & Second-order PML method & Hybrid ABC method \\
\hline $\begin{array}{l}\text { Homogeneous model } \\
\text { PML }=10\end{array}$ & $20 \mathrm{~s}$ & $28 \mathrm{~s}$ & $24 \mathrm{~s}$ & $20 \mathrm{~s}$ \\
\hline $\begin{array}{l}\text { Homogeneous model } \\
\text { PML }=20\end{array}$ & $24 \mathrm{~s}$ & $34 \mathrm{~s}$ & $28 \mathrm{~s}$ & $25 \mathrm{~s}$ \\
\hline $\begin{array}{l}\text { Constant-gradient velocity model } \\
\text { PML }=10\end{array}$ & $21 \mathrm{~s}$ & $30 \mathrm{~s}$ & $26 \mathrm{~s}$ & $21 \mathrm{~s}$ \\
\hline $\begin{array}{l}\text { Constant-gradient velocity model } \\
\mathrm{PML}=20\end{array}$ & $25 \mathrm{~s}$ & $35 \mathrm{~s}$ & $30 \mathrm{~s}$ & $26 \mathrm{~s}$ \\
\hline $\begin{array}{l}\text { Marmousi model } \\
\text { PML }=10\end{array}$ & $6 \mathrm{~m} 57 \mathrm{~s}$ & $10 \mathrm{~m} 31 \mathrm{~s}$ & $8 \mathrm{~m} 20 \mathrm{~s}$ & $7 \mathrm{~m} 14 \mathrm{~s}$ \\
\hline $\begin{array}{l}\text { Marmousi model } \\
\mathrm{PML}=20\end{array}$ & $6 \mathrm{~m} 05 \mathrm{~s}$ & $9 \mathrm{~m} 05 \mathrm{~s}$ & $7 \mathrm{~m} 18 \mathrm{~s}$ & $6 \mathrm{~m} 25 \mathrm{~s}$ \\
\hline
\end{tabular}

parison, we also calculated the value of the reflection coefficient $R$ using Eq. (13) and plotted the corresponding curve in Fig. 13.

As can be seen in Figs. 12 and 13, all of the four methods can absorb the reflected waves to a certain degree. For the same number of absorbing layers, the absorption performance of our method and that of the classic SG PML method are almost the same and both methods are superior to the other two methods, while the hybrid ABC method is the worst. Specifically, when the number of absorbing layers equals 10 , the absorption coefficients of our method and classic SG PML method are both $-60 \mathrm{~dB}$, which means that the amplitude of the reflected wave after absorbing is only $0.1 \%$ of that before absorbing. Increasing the number of absorbing layers can improve the absorption effect of the four methods. In addition, the 20-layer second-order PML method performs similarly to the 10-layer proposed method and the 10-layer classic SG PML method. This indicates that the second-order PML method always requires more absorbing layers than the first-order PML does. Although the core idea of the secondorder PML method is the same as that of the first-order PML method, there are very different ways to deal with mathematical equations. In the second-order method, the original PML equations are transformed and some additional nonphysical variables are added, which greatly reduces the efficiency of boundary conditions. Moreover, the second-order PML method still needs to handle the first-order system, i.e., a Euler or a Runge-Kutta time scheme has to be used (Komatitsch and Tromp, 2003). So, in this case, the second-order PML method is naturally less effective than our first-order PML method and requires more absorbing layers. For hybrid $\mathrm{ABC}$, it has poor absorbing performance compared to the PML method since it is based on a one-way equation. So, it would have limited improvement due to the phase and the amplitude errors that are associated with the expansion of one-way wave equation.

\subsubsection{Constant-gradient velocity model}

Taking into account the fact that the homogeneous model is relatively simple and quite different from the actual distribution of an underground medium, the second model that we use is the constant-gradient velocity model, as shown in Fig. 14. The velocity is $1500 \mathrm{~m} \mathrm{~s}^{-1}$ at $z=0 \mathrm{~m}$ and $3500 \mathrm{~m} \mathrm{~s}^{-1}$ at $z=2000 \mathrm{~m}$, and the velocity gradient is 1 . The source and receiver locations are exactly the same as those in the first homogeneous model. Figures 15 and 16 compare the receiver records and the reflection coefficients $R$ of the four methods for different boundary conditions. It can be seen that the absorption effect of this model is not as good as that of the homogeneous model, but the results are the same. For the same number of absorbing layers, our method has the same absorbing ability as that of the classic SG PML and performs better than the other two methods. Also, we still need to use more layers for the second-order PML method instead of using the thin method we proposed. In addition, we see that the 10-layer proposed method is much better than the 20-layer hybrid ABC method.

\subsubsection{Marmousi model}

We next compared the absorption efficiency of the four methods for a complex Marmousi model. The Marmousi model has a size of $9200 \mathrm{~m} \times 3000 \mathrm{~m}$, a grid spacing of $12.5 \mathrm{~m}$, a time step of $0.001 \mathrm{~s}$, and a total recording time of $8 \mathrm{~s}$. The velocity distribution is shown in Fig. 17. Taking the first shot of the Marmousi model as an example, we can see that the shot is located on the ground surface at a horizontal distance of $3000 \mathrm{~m}$ and that the 185 receivers are evenly distributed between 0 and $9200 \mathrm{~m}$ on the surface. The results in Fig. 18 show that the absorption effect of our method is equal to or better than the absorption effect of the other methods. When the number of PMLs is 20, the reflected wave is relatively small. Therefore, the method we propose is also suitable for simulating complex models. 
Based on the above numerical experiments, although the hybrid ABC method is often used as the boundary condition of the CG-based method because it is easy to deduce its second-order form, its absorption performance is obviously worse than that of the other three PML methods since it is based on a one-dimensional wave equation. Among the three PML methods, the 10-layer classic SG PML method (firstorder PML is used inside) for the first-order wave equation is enough to suppress the edge reflections, while the 20-layer second-order PML method is sufficient for the second-order wave equation. However, our first-order PML method only requires a thickness of 10 grid spacings to absorb the outgoing wave entirely. It may have a significant advantage over the second-order PML method. Table 2 shows the computation times of the four methods for different numbers of absorbing layers. Among them, the computation time of our method is the shortest and that of the classic SG PML method is the longest. Given that our method uses the CG scheme in the computational area, it requires much less computation time than the classic SG PML method does. In addition, the second-order PML method requires the transformation of the original first-order PML equation into a second-order form. The required complex formulas and extra variables without physical meaning increase the computation time. In addition, our method naturally implements high-order temporal discretization if necessary, while the second-order PML method does not. Therefore, our method is ideal for seismic wave forward modeling.

\subsection{Three-dimensional homogeneous model}

In order to facilitate the experiments and comparative analyses, we used the two-dimensional models described in the above numerical experiments. To further illustrate the effectiveness of our method, Fig. 19 shows the experimental results of this method for a threedimensional homogeneous velocity model. The model size is $1000 \mathrm{~m} \times 1000 \mathrm{~m} \times 1000 \mathrm{~m}$, the grid spacing is $10 \mathrm{~m}$, and the velocity is $2000 \mathrm{~m} \mathrm{~s}^{-1}$. The source is located at $(500,500$, $500 \mathrm{~m}$ ) with a time step of $0.001 \mathrm{~s}$. Figure 19 shows snapshots of the wave field at different times. From this, we find that when the number of PMLs is 20, the wave field record is very clear, and almost no reflected waves are seen.

\section{Conclusions}

We propose a new boundary-matched algorithm that effectively combines the CG scheme in the computational area and the SG scheme in the PML boundary conditions, while preserving the high computational efficiency of the CG scheme and the good absorption effect of PML boundary conditions. Our proposed method is easy to implement, and we only perform appropriate wave field matching at the grid points, which avoids complicated modifications to the PML formulas and the introduction of unnecessary variables. The numerical experiments of the different models indicate that our method is applicable to a variety of simple and complex two-dimensional and three-dimensional geological models. For the same conditions, our method can achieve similar or better accuracy and reflected wave absorption efficiency compared to other boundary absorption methods, while it requires less computation time. Because our method keeps the independence of the computational area and the boundary absorption area, it can also be combined with other CG-based seismic wave numerical algorithms, such as the nearly analytical center difference method with PML boundary conditions, to achieve better numerical simulation accuracy.

Our work is based on the numerical simulation of a scalar equation. Because the elastic wave equation includes more wave field information, it is also widely used in the numerical simulation of seismic waves. The simulation of the elastic wave equation requires more computations and greater storage capacity, while our proposed method can reduce the computational cost. The next step of our work will be the numerical simulation of the elastic wave equation and is expected to significantly improve its computational efficiency.

Data availability. The processed data required to reproduce these findings are available to download from https://doi.org/10.17632/wv6kwbmf2h.1 (Zhang, 2018).

Author contributions. XZ and QC contributed to the conception of the study. XZ, DZ, and YY contributed significantly to analysis and manuscript preparation; XZ, DZ, and QC performed the data analyses and wrote the manuscript; XZ and DZ helped perform the analysis with constructive discussions.

Competing interests. The authors declare that they have no conflict of interest.

Special issue statement. This article is part of the special issue "Advances in seismic imaging across the scales". It does not belong to a conference.

Acknowledgements. This research work was financially supported by the National Science and Technology Major Project of China under grant 2011zx05003-003.

Edited by: Michal Malinowski

Reviewed by: two anonymous referees 


\section{References}

Alford, R. M., Kelly, K. R., and Boore, D.: Accuracy of finitedifference modeling of the acoustic wave equation, Geophysics, 39, 834-842, 1974.

Berenger, J. P.: A Perfect Matched Layer for the Absorption of Electromagnetic Waves, J. Comput. Phys., 114, 185-200, 1994.

Blanch, J. O. and Robertsson, J. O. A.: A modified Lax-Wendroff correction for wave propagation in media described by Zener elements, Geophys. J. Roy. Astr. S., 131, 381-386, 2010.

Cerjan, C., Kosloff, D., Kosloff, R., and Reshef, M.: A nonreflecting boundary condition for discrete acoustic and elastic wave equations, Geophysics, 50, 705-708, 1985.

Cerveny, V.: Seismic ray theory, Cambridge University Press, 2001.

Chew, W. C. and Liu, Q. H.: Perfectly matched layers for elastodynamics: a new absorbing boundary condition, J. Comput. Acoust., 4, 341-359, 1996.

Chu, C. L. and Stoffa, P. L.: Determination of finite-difference weights using scaled binomial windows, Geophysics, 77, W17W26, 2012.

Clayton, R. and Engquist, B.: Absorbing boundary conditions for acoustic and elastic wave equations, B. Seismol. Soc. Am., 67, 1529-1540, 1977.

Collino, F. and Tsogka, C.: Application of the perfectly matched absorbing layer model to the linear elastodynamic problem in anisotropic heterogeneous media, Geophysics, 66, 294-307, 1998.

Dablain, M. A.: The application of high-order differencing to the scalar wave equation, Geophysics, 51, 54, https://doi.org/10.1190/1.1442040, 1986.

Dan, D. K. and Baysal, E.: Forward modeling by a Fourier method, Geophysics, 47, 1402-1412, 1982.

Engquist, B. and Runborg, O.: Computational high frequency wave propagation, Acta Numer., 12, 181-266, 2003.

Fornberg B.: High-Order Finite Differences and the Pseudospectral Method on Staggered Grids, Siam J. Numer. Anal., 27, 904-918, 1990.

Gao, Y. J., Song, H., Zhang, J., and Yao, Z.: Comparison of artificial absorbing boundaries for acoustic wave equation modelling, Explor. Geophys., 48, 76-93, 2017.

Gold, N., Shapiro, S. A., and Burr, E.: Modelling of high contrasts in elastic media using a modified finite difference scheme, Seg Technical Program Expanded, 1850-1853, 1997.

Grote, M. J. and Sim, I.: Efficient PML for the wave equation, Mathematics, available at: http://arxiv.org/abs/1001.0319v1 (last access: 30 July 2015), 2010

Higdon, R. L.: Absorbing boundary conditions for elastic waves, Siam J. Numer. Anal., 31, 64-100, 2012.

Huang, C. and Dong, L.: Staggered-Grid High-Order FiniteDifference Method in Elastic Wave Simulation with Variable Grids and Local Time Steps, Chinese J. Geophys., 52, 1324$1333,2009$.

Hustedt, B., Operto, S., and Virieux, J.: Mixed-grid and staggeredgrid finite-difference methods for frequency-domain acoustic wave modelling, Geophys. J. Roy. Astr. S., 157, 1269-1296, 2004.

Igel, H., Mora, P, and Riollet, B.: Anisotropic wave propagation through finite-difference grids, Geophysics, 60, 1203-1216, 1995.
Kelly, K. R., Ward, R. W. Treitel, S., and Alford, R. M.: Synthetic seismograms: a finite-difference approach, Geophysics, 41, 2 27, 2012.

Komatitsch, D. and Martin, R.: An unsplit convolutional perfectly matched layer improved at grazing incidence for the seismic wave equation, Geophysics, 72, SM155-SM167, 2007.

Komatitsch, D. and Tromp, J.: A perfectly matched layer absorbing boundary condition for the second-order seismic wave equation, Geophys. J. Int., 154, 146-153, 2003.

Kreiss, H. O. and Oliger, J.: Comparison of accurate methods for the integration of hyperbolic equations, Tellus, 24, 199-215, 1972.

Lax, P. D. and Wendroff, B.: Difference schemes for hyperbolic equations with high order of accuracy, Commun. Pure Appl. Math., 17, 381-398, 1964.

Li, B., Liu, Y., Sen, M. K., and Ren, Z.: Time-space-domain meshfree finite difference based on least squares for 2D acoustic-wave modeling, Geophysics, 82, 143-157, 2017.

Liu, Q. H. and Tao, J.: The perfectly matched layer for acoustic waves in absorptive media, J. Acoust. Soc. Am., 102, 2072-2082, 1998.

Liu, Y. and Sen, M. K.: Scalar Wave Equation Modeling with Time-Space Domain Dispersion-Relation-Based Staggered-Grid Finite-Difference Schemes, B. Seismol. Soc. Am., 101, 141-159, 2011.

Madariaga, R.: Dynamics of an expanding circular fault, B. Seismol. Soc. Am., 66, 639-666, 1976.

Marfurt, K. J.: Accuracy of finite-difference and finite-element modeling of the scalar and elastic wave equations, Geophysics, 49, 533-549, 1987.

Moczo, P. Robertsson, J. O. A., and Eisner, L.: The FiniteDifference Time-Domain Method for Modeling of Seismic Wave Propagation, Adv. Geophys., 48, 421-516, 2007.

Moczo, P., Kristek, J., Galis, M., Chaljub, E., and Etienne, V.: 3 D finite-difference, finite-element, discontinuous-Galerkin and spectral-element schemes analysed for their accuracy with respect to $P$-wave to $S$-wave speed ratio, Geophys. J. Int., 187, 1645-1667, 2011

Moczo, P., Kristek, J., and Galis, M.: The finite-difference modelling of earthquake motions: waves and ruptures, Cambridge University Press, 1-365, 2014.

O'Brien, G. S.: 3D rotated and standard staggered finite-difference solutions to Biot's poroelastic wave equations: Stability condition and dispersion analysis, Geophysics, 75, 111-119, 2010.

Pasalic, D. and McGarry, R.: Convolutional perfectly matched layer for isotropic and anisotropic acoustic wave equations, 2010 SEG Annual Meeting, Society of Exploration Geophysicists, 2010.

Ren, Z. M. and Liu, Y.: A Hybrid Absorbing Boundary Condition for Frequency-Domain Visco-Acoustic Finite-Difference Modeling, J. Geophys. Eng., 10, 86-95, 2012.

Reynolds, A. C.: Boundary conditions for the numerical solution of wave propagation problems, Geophysics, 43, 155-165, 1978.

Saenger, E. H., Gold, N., and Shapiro, S. A.: Modeling the propagation of elastic waves using a modified finite-difference grid, Wave Motion, 31, 77-92, 2000.

Sánchez-Sesma, F. J. Madariaga, R., and Irikura, K.: An approximate elastic two-dimensional Green's function for a constantgradient medium, Geophys. J. Int., 146, 237-248, 2001. 
Sochacki, J, Kubichek, R., George, J., Fletcher, W., and Smithson, S.: Absorbing boundary conditions and surface waves, Geophysics, 52, 60-71, 1987.

Tan, S. R. and Huang, L.: A staggered-grid finite-difference scheme optimized in the time-space domain for modeling scalar-wave propagation in geophysical problems, J. Comput. Phys., 276, 613-634, 2014.

Virieux, J.: SH-wave propagation in heterogeneous media: Velocity-stress finite-difference method, Geophysics, 49, 19331942, 1984.

Virieux, J.: P-SV wave propagation in heterogeneous media; velocity-stress finite-difference method, Geophysics, 49, 19331942, 1986.

Wang, S. D.: Absorbing boundary condition for acoustic wave equation by perfectly matched layer, Oil Geophysical Prospecting, 38, 31-34, 2003.

Yang, D. Liu, E. Zhang, Z. J. and Teng, J.: Finite-difference modelling in two-dimensional anisotropic m,edia using a fluxcorrected transport technique, Geophys. J. Int., 148, 320-328, 2002.

Yang, D., Liu, E., and Zhang, Z.: Evaluation of the u-W finite element method in anisotropic porous media, J. Seism. Explor., 17, 273-299, 2008.
Yang, D. H., Teng, J. W., Zhang, Z. J., and Liu, E.: A nearly analytic discrete method for acoustic and elastic wave equations in anisotropic media, B. Seismol. Soc. Am., 93, 2389-2401, 2003.

Yang, D. H., Tong, P., and Deng, X.: A central difference method with low numerical dispersion for solving the scalar wave equation, Geophys. Prospect., 60, 885-905, 2012.

Yang, L., Yan, H., and Liu, H.: Optimal rotated staggered-grid finite-difference schemes for elastic wave modeling in TTI media, J. Appl. Geophys., 122, 40-52, 2015.

Zhang, J. H. and Yao, Z. X.: Optimized finite-difference operator for broadband seismic wave modeling, Geophysics, 78, A13-A18, 2013.

Zhang, X.: Seismic dataset of different finite difference method and different absorbing conditions, Mendeley Data, https://doi.org/10.17632/wv6kwbmf2h.1, 2018.

Zhang, Z. G., Zhang, W., Li, H., and Chen, X.: Stable discontinuous grid implementation for collocated-grid finite-difference seismic wave modelling, Geophys. J. Int., 192, 1179-1188, 2013. 